\title{
2 Kompilation und Autorschaft
}

Die Kompilation war nicht nur die wichtigste Textproduktionspraktik der Weckerschen Buchproduktion, sie war auch eine für die Frühe Neuzeit insgesamt typische Praktik der Text- und Bildproduktion. Sie findet sich bei enzyklopädisch ausgerichteten Werken wie etwa Pandectae, Theatri oder Bibliothecae, bei Tierbüchern, Kosmographien, Herbarien, Chronographien oder Florilegiensammlungen sowie bei weniger systematisch arrangierten Veröffentlichungen der Buntschriftstellerei, bei Flugblättern, Briefsummarien und Nachrichtensammlungen. ${ }^{185}$ Die Kompilation zeichnet sich dadurch aus, dass Text- oder Bildelemente bestehenden Veröffentlichungen entnommen, wiederverwendet und durch eine neue Ordnung sowie Kontextualisierung zu etwas Neuem verarbeitet wurden. ${ }^{186}$ Dabei ist die Kompilation nach Volker Hertel „eine grundlegende, lange tradierte Form der Wissensaneignung, der Wissensverarbeitung (einschließlich der Wissenssystematisierung) und der Wissensvermittlung. “187 Jedoch herrscht laut Susanne Kaup in den Literaturwissenschaften Uneinigkeit darüber, was unter einer Kompilation genau zu verstehen ist. Handelt es sich um eine Gattung, eine Form des Schreibens oder aber um den Prozess, in Büchern fixiertes Wissen zugänglich zu machen? Einigkeit besteht nach Kaup nur darin, dass es sich um strukturierte Textsammlungen handelt. ${ }^{188}$ All diese Elemente sind meiner Meinung nach Teil der Kompilation, da diese durch die dahinterstehenden Praktiken entsteht, weshalb das eine das andere nicht ausschliesst.

185 Siehe u.a. Zedelmaier: Navigieren im Textuniversum, 2008, insbes. S. 114; Schierbaum: Enzyklopädistik 1550 -1650, 2009; Stammen, Weber, Wolfgang E. J: Wissenssicherung, Wissensordnung und Wissensverarbeitung, 2004; Schock, Flemming: Wissensliteratur und ,Buntschriftstellerei' in der Frühen Neuzeit, 2012, S. 1-20, hier S. 3f.; Schock: Polyhistorismus und Buntschriftstellerei, 2012; Harms, Schilling: Das illustrierte Flugblatt der frühen Neuzeit, 2008; Schilling: Flugblatt und Drama in der Frühen Neuzeit, 2008, u.a. S. 243f.; Zwierlein: Fuggerzeitungen als Ergebnis von italienisch-deutschem Kulturtransfer 1552-1570, 2010, S. 177. Dieser Abschnitt basiert auf: Zweifel: Ein Blick hinter die Produktion von Kompilationen im 16. Jahrhundert am Beispiel Johann Jacob Weckers, 2018.

186 Vgl. Kaup: De beatitudinibus, 2012, S. 129f.

187 Hertel, Volker: Kompilieren, Klassifizieren, Contrafactieren. Aspekte gelehrter Textproduktion in der Frühen Neuzeit, in: Wiese, Ingrid; Barz, Irmhild; Fix, Ulla (Hg.): Fachtextsorten, gestern und heute. Ingrid Wiese zum 65. Geburtstag (= Leipziger Arbeiten zur Sprach- und Kommunikationsgeschichte). Frankfurt am Main: Peter Lang, 2008, S. 27-46, hier S. 7; Waiter, Nicolas: Geschichtsschreibung und Kompilation: Diodors historiographische Arbeitsmethode und seine Vorstellungen von zeitgemäßer Geschichtsschreibung, in: Rheinisches Museum 149, 2006, S. $248-271$.

188 Kaup: De beatitudinibus, 2012, S. 130.

Ә OpenAccess. (c) 2022 Simone Zweifel, publiziert von De Gruyter. (cc)/BY Dieses Werk ist lizenziert unter einer Creative Commons Namensnennung 4.0 International Lizenz. https://doi.org/10.1515/9783110740516-003 
Die Textproduktionspraktik der Kompilation erlaubte es, unterschiedlichste Textelemente zusammenzutragen. Dies wird sichtbar bei den Publikationen Johann Jacob Weckers. Sie enthalten eine grosse Vielfalt an Wissensbeständen, auch solchen, die aus gewissen Forschungsperspektiven klar voneinander getrennt waren, wie beispielsweise die Zuteilung von Personen zu Galenisten respektive Paracelsisten. ${ }^{189}$ Diese Trennung wird hinterfragt, betrachtet man die Secreti Weckers, in denen sich sowohl Rezepte, die Galen, als auch solche, die Paracelsus zugeschrieben werden, finden. ${ }^{190}$ Das Zusammenbringen solch unterschiedlicher Wissensbestandteile erlaubte es, neue Perspektiven zu gewinnen, wodurch neue Erkenntnisse und folglich auch neues Wissen generiert werden konnten. ${ }^{191}$ Für Lesende war es zudem dank der Form der Kompilation relativ einfach, an diese Wissensbestände zu gelangen und diese zu rezipieren. ${ }^{192}$ Dies mag zum Erfolg frühneuzeitlicher Kompilationen beigetragen haben, der unter anderem an der grossen Anzahl Publikationen von Conrad Gessner, Walther Hermann Ryff, Theodor Zwinger und auch Johann Jacob Wecker sichtbar wird. ${ }^{193}$

189 Zur Vielfalt dieser Wissensbestände siehe auch Kavey: Books of Secrets, 2007, S. 33; Martins: Les livres de secrets imprimés et traduits en Europe, 2015, S. 146.

190 Wecker: De Secretis libri XVII, 1582, passim. Auch Tillmann Walter hinterfragt die strikte Trennung zwischen Paracelsisten und Galenisten: Ders.: New Light on Antiparacelsianism, 2012. Von einer partiellen Vermischung geht auch Hans-Peter Neumann aus: Ders.: Wissenspolitik in der frühen Neuzeit am Beispiel des Paracelsismus, in: Jaumann, Herbert (Hg.): Diskurse der Gelehrtenkultur in der Frühen Neuzeit. Ein Handbuch. Berlin: De Gruyter, 2011, S. 255-304, hier S. $265 \mathrm{f}$. Er fagt gar: „Ist das proklamierte `Neue` und `Andere` der Paracelsistischen Wissenschaft eventuell nur Resultat einer Neugier und Interesse weckenden Propagandastrategie, um die Paracelsistische Medizin von der vermeintlichen Obsoletheit der galenistischen Konkurrenz abzusetzen und ins rechte Licht zu rücken?“ Ebd., S. 263. Zu Paracelsismus und Galenismus siehe insbesondere Kühlmann, Telle: Corpus Paracelsisticum: Band II; Walter, New Light on Antiparacelsianism, 2012.

191 Vgl. Hertel: Kompilieren, Klassifizieren, Contrafactieren, 2008, S. 3f. Siehe auch: Heß: Enzyklopädien und Florilegien im 16. und 17. Jahrhundert, 2004, S. 49f; Blair: Too Much to Know, 2010, S. 177.

192 Vgl. Kaup: De beatitudinibus, 2012, S. 137.

193 Siehe dazu u.a. Blair: Too Much to Know, 2010; Leu, Urs B.: Conrad Gessner (1516-1565). Universalgelehrter und Naturforscher der Renaissance (= NZZ Libro). Zürich: Verlag Neue Zürcher Zeitung, 2016; Carlino: Kunstbüchlein and Imagines Contrafactae, 2008; Kranich-Hofbauer: Walther Hermann Ryff, 2012; Zedelmaier: Navigieren im Textuniversum, 2008. 


\subsection{Autorschaft von Kompilationen und „geistiges Eigentum“}

Kompiliert wurde schon in der Antike, Beispiele dafür sind die Bibliotheke des Diodor sowie die Historia naturalis (Buch 12-27) des Plinius. ${ }^{194}$ Es kann angenommen werden, dass schon vorher und auch in anderen kulturellen Kontexten unterschiedliche Text- und Bildelemente $\mathrm{zu}$ Neuem verarbeitet wurden und werden. So wurde die Technik der Kompilation etwa im Mittelalter in verschiedenen Bereichen - sowohl in rechtlichen, historiographischen, philosophischen und theologischen - angewendet. ${ }^{195}$ Ungefähr im 13. Jahrhundert kann laut Alastair J. Minnis beobachtet werden, dass die Kompilatoren ein eigenes literarisches Bewusstsein entwickelten, bei dem sie die Autorität auf die Verfassenden abwälzten, von denen sie kompiliert hatten. ${ }^{196}$ Folglich seien sie als Kompilatoren zwar für Auswahl und Zusammenstellung des Bestehenden verantwortlich gewesen, nicht aber für die Inhalte selbst. Die Verantwortung über letztere sei den autores aufgrund ihrer auctoritas (Autorität) zugekommen. ${ }^{197}$ So erklärte etwa Vincent de Beauvais (ca. 1190 -1264) in seinem 1247 oder 1260 abgeschlossenen Speculum maius, dass das Werk nicht nur von ihm sei, sondern dass er es vielmehr aus bestehenden Aussagen zusammengefügt habe. Autorisiert würde es von den Autoritäten, deren Texte er zusammengestellt habe; von ihm stamme nur die Anordnung der Teile. ${ }^{198}$ Eine sehr ähnliche Erklärung findet sich in der Historiae figuralis des Girardus de Avernia 1272. Er schrieb, dass er kaum etwas oder quasi nichts zu diesem Werk hinzugefügt habe, dessen Autorität bei Vielen liege. Sein Anteil habe lediglich darin bestanden, die Teile zusammenzustellen und den Text

194 Siehe Hertel: Kompilieren, Klassifizieren, Contrafactieren, 2008, S. 3. Zur Kompilation in der Antike siehe u. a. Natili, Daniele: „Justiniani Digesta“. Methode und Quellen der Kompilation, in: Piccione, Rosa Maria; Perkams, Matthias (Hg.): Selecta colligere, I. Akten des Kolloquiums „Sammeln, Neuordnen, Neues Schaffen. Methoden der Überlieferung von Texten in der Spätantike und in Byzanz“ (Jena, 21.-23. November 2002) (= Hellenica). Alessandria: Ed. dell’Orso, 2003-2005, S. 117-141; Waiter: Geschichtsschreibung und Kompilation, 2006.

195 Kaup: De beatitudinibus, 2012, S. 127. Zu mittelalterlichen Kompendien siehe Steiner, Emily: Introduction, in: Steiner, Emily; Ransom, Lynn (Hg.): Taxonomies of Knowledge. Information and Order in Medieval Manuscripts (= The Lawrence J. Schoenberg Studies in Manuscript Culture). Philadelphia: University of Pennsylvania Press, 2015, S. 1-6, hier S. 1.

196 Minnis, Alastair J.: Late-Medieval Discussions of Compilatio and the Rôle of the Compilator, in: Beiträge zur Geschichte der deutschen Sprache und Literatur 101, 1979, S. 385-421, hier S. 387. 197 Ebd.

198 „,...] hoc ipsum opus utique meum simpliciter non sit, sed illorum potius ex quorum dictis fere totum illud contextui. Nam ex meo pauca, vel quasi nulla addidi. Ipsorum igitur est auctoritate, nostrum autem sola partium ordinatione." De Beauvais, Vincent: >Speculum maius<, apologia actoris, cap. III, Dijon MS 568. Dieses Zitat stammt von ebd., S. 387. Übersetzung und Paraphrasierung: Simone Zweifel und Jodok Trösch. 
auszugestalten. ${ }^{199}$ Beide Autoren respektive Kompilatoren argumentierten folglich, dass die Autorität der Texte nicht bei den Kompilierenden, also bei ihnen selbst, liege, sondern bei den auctores. ${ }^{200}$ Letztere waren nicht aktiv in die Textproduktion involviert, vielmehr waren es die von ihnen hinterlassenen oder ihnen zugeschriebenen Bücher, die in die Texte einflossen. Dabei wurden Wissenselemente der Autoritäten kompiliert, nicht aber kritisiert: „[...] Kritik hätte die Geltung der Aussagen gemindert. Die Aussagen der Autoritäten sind das Mittel, den eigenen Punkt stark zu machen und zugleich sich zu schützen“, schreibt Martin Gierl. ${ }^{201}$ Auch überliessen die Kompilierenden das Urteil über „richtig“ und „falsch“ den Lesenden, da sie schliesslich „nur“ geordnet und zusammengeschrieben hatten und weder für die Verständlichkeit noch für mögliche Missverständnisse verantwortlich waren. ${ }^{202}$

Neben der Vorstellung des Kompilators finden sich im Mittelalter, zumindest bei Bonaventura, auch jene des Autors, des Kommentators und des Schreibers, wobei das Unterscheidungsmerkmal „der jeweils abnehmende Eigenanteil“ darstelle und der Kompilator an zweiter Stelle genannt wird. ${ }^{203}$ Inwiefern diese

199 De Avernia, Girardus: Historiae figuralis, um 1272, nach Delisle, Leopold: Le chroniqueur Girard d'Auvergne ou d'Anvers, in: Journal des Savants, 1900, S. 232-243 sowie S. 285-294, hier S. 235. Dazu: Melville, Gert: Kompilation, Fiktion und Diskurs. Aspekte zur heuristischen Methode der mittelalterlichen Geschichtsschreiber, in: Meier, Christian; Rüsen, Jörn (Hg.): Historische Methode. München 1988 (= Theorie der Geschichte), S. 133-153, hier S. 134. Siehe auch Gierl, Martin: Kompilation und die Produktion von Wissen im 18. Jahrhundert, in: Zedelmaier, Helmut; Mulsow, Martin (Hg.): Die Praktiken der Gelehrsamkeit in der Frühen Neuzeit (= Frühe Neuzeit, 64). Tübingen: De Gruyter, 2001, S. 63-94, hier S. 66.

200 Vgl. ebd., S. 67. Diese Autoritäten standen laut Anne Coldiron auch für die geforderte Akkuratheit und die Wahrhaftigkeit des Inhalts: Coldiron, Anne: Women in Early English Print Culture, in: Bicks, Caroline; Summit, Jennifer (Hg.): The History of British Women's Writing, 1500 1620. Volume Two (= History of British Women's Writing). London: Palgrave Macmillan, 2010, S. $60-83$, hier S. 61 .

201 Gierl: Kompilation und die Produktion von Wissen im 18. Jahrhundert, 2001, S. 67. Vgl. Minnis: Late-Medieval Discussions of Compilatio and the Rôle of the Compilator, 1979, S. $418 \mathrm{ff}$. 202 Dies gilt auch für das 16. Jahrhundert: Blair: Too Much to Know, 2010, S. 177. Siehe auch Minnis: Late-Medieval Discussions of Compilatio and the Rôle of the Compilator, 1979, S. 389-391. 203 Suerbaum, Almut: Accessus ad auctores: Autorkonzeptionen in mittelalterlichen Kommentartexten, in: Andersen, Elizabeth; Haustein, Jens; Simon, Anne u. a. (Hg.): Autor und Autorschaft im Mittelalter. Kolloquium Meißen 1995. Tübingen: De Gruyter, 1998, S. 29-37, hier S. 30. Sie bezieht sich auf Bonaventura: Commentaria in IV libros Sententiarum Magistri Petri Lombardi, Prooemium, quaest IV, conclusio, in: Opera omnia, Bd. 1. Quaracchi, 1892, S. 14f.: „Ad intelligentiam dictorum notandum, quod quadruplex est modus faciendi librum. Aliquis enim scribit aliena, nihil addendo vel mutando; et iste mere dicitur scriptor. Aliquis scribit et aliena, addendo, sed non de suo; et iste ,compilator dicitur. Aliquis scribit et aliena et sua, sed aliena tamquam principalia, et sua tamquam annexa ad evidentiam; et iste dicitur ,commentator', non auctor. 
Ausdifferenzierung als allgemeingültig betrachtet werden kann, muss an dieser Stelle offenbleiben. Klar ist jedoch, dass im Mittelalter - wie auch schon in der Antike - eine Idee von Autorschaft existent war, wenn auch nicht gekoppelt an jene des „geistigen Eigentums“. ${ }^{204}$ Eine Autorschaftsvorstellung findet sich auch in den Weckerschen Publikationen, in denen von „authores“ gesprochen wird, dies jedoch unabhängig davon, ob sie selbst Text verfassten oder kompilierten. ${ }^{205}$ Damit ist die von Bonaventura getroffene Ausdifferenzierung von Autor, Kompilator, Kommentator und Schreiber hier nicht relevant: sie scheint schlicht nicht gemacht worden zu sein.

Frühneuzeitliche Autorschaft zeigt sich nach Karl A. E. Enenkel insbesondere in Vorreden und Widmungsschreiben, bei denen sich der Autor ,wie ein Firmenlogo und -Emblem, wie eine Reklamesäule vor den Text“" gestellt habe. ${ }^{206}$ Diese Vorstellung kann auf die Autorschaft von Kompilationen übertragen werden, wenn man sich auf die Person bezieht, die auf dem Titelblatt genannt ist. Nach Martha Woodmansee ist die frühneuzeitliche Autorschaft aufgeteilt in zwei Arten: Einerseits habe es die Autoren und Autorinnen als Handwerker und Handwerkerinnen gegeben, anderseits die „inspirierten“ Autorinnen und Autoren, deren Inspiration entweder von den bestehenden Strukturen oder von Gott gekommen sei. ${ }^{207}$ Bei beiden ist nicht der Autor oder die Autorin persönlich für das Geschriebene verantwortlich, vielmehr steckten entweder strukturelle oder

Aliquis scribit et sua et aliena, sed sua tanquam principalia, aliena tamquam annexa ad confirmationem; et talis debet dici ,auctor" "Siehe auch Minnis, Alastair J.: Medieval Theory of Authorship. Scholastic Literary Attitudes in the Later Middle Ages. London: Scolar Press, 1984, S. 94 f.; Cook, Trevor J. N.: Plagiarism and Proprietary Authorship in Early Modern England, 1590 1640, University of Toronto, Department of English. Toronto, 2011, S. 34 f.; Eisenstein, Elizabeth L.: The Printing Revolution in Early Modern Europe. Cambridge: Cambridge Univ. Press, 2005², S. 95. $204 \mathrm{Zu}$ Autorschaftsvorstellungen in Antike und Mittelalter siehe u. a. Calame, Claude; Chartier, Roger (Hg.): Identités d'auteur dans l'antiquité et la tradition européenne (= Collection HOROS). Grenoble: Jérôme Millon, 2004; Calame, Claude: Identités d'auteur à l'exemple de la Grèce classique: signatures, énonciations, citations, in: Ders.; Chartier, Roger (Hg.): Identités d'auteur dans l'antiquité et la tradition européenne (= Collection HOROS). Grenoble: Jérôme Millon, 2004, S. 11-39; Minnis, Alastair J.: Medieval Theory of Authorship. Scholastic Literary Attitudes in the Later Middle Ages. Aldershot: Scolar Press, $1988^{2}$; Andersen, Elizabeth; Haustein, Jens; Simon, Anne u.a. (Hg.): Autor und Autorschaft im Mittelalter. Kolloquium Meißen 1995. Tübingen: De Gruyter, 1998; Ingold, Felix Philipp; Wunderlich, Werner (Hg.): Der Autor im Dialog. Beiträge zu Autorität und Autorschaft. St. Gallen: UVK, 1995; Bolens, Guillemette; Erne, Lukas (Hg.): Medieval and Early Modern Authorship (= Swiss Papers in English Language and Literature). Tübingen: Narr, 2011.

205 Siehe zum Beispiel die Liste der „authores“ der Wecker: De Secretis libri XVII, 1582.

206 Enenkel: Die Stiftung von Autorschaft in der neulateinischen Literatur, 2015, S. 17.

207 Woodmansee: The Author, Art, and the Market, 1994, S. 36. 
inspiratorische Kräfte dahinter. ${ }^{208}$ Im 18. Jahrhundert sei der Aspekt des Handwerkertums in den Hintergrund getreten; die „inspirierte“ Autorschaft habe sich verlagert: „,Inspiration“ came to be explicated in terms of original genius, with the consequence that the inspired work was made peculiarly and distinctively the product - and the property - of the writer."209 Dabei bezieht sie sich auf die folgende Formulierung über die Sprache der Dichter in der Theorie der schönen Künste von Johann Gregor Sulzer aus dem Jahr 1798: „[...] deßwegen sie [die Sprache der Dichter, SZ] auch eine außerordentliche Veranlassung haben muß, welche ohne Zweifel in dem Genie und Charakter des Dichters zu suchen ist."210 Diese zwei Arten der Autorschaft waren meines Erachtens nicht so strikte voneinander getrennt, wie dies von Woodmansee angenommen wird. Vielmehr gehe ich davon aus, dass sie ineinander übergehen konnten. Dies zeigt das Beispiel Weckers, der sowohl handwerklicher Autor als auch von bestehenden Texten und Strukturen geprägt war. Hinzu kommt, was gerade bei der Weckerschen Textproduktion der Fall ist, dass eine Autorpersönlichkeit in der Frühen Neuzeit oft nicht alleine für den produzierten Text verantwortlich war: Meist waren mehrere Akteurinnen und Akteure sowie Elemente in die Herstellung eines Textes involviert, der oft einer Person zugeschrieben wurde. ${ }^{211}$ Diese Personen agierten Buchpraktiken aus; die Autorschaft kann als Mantel dieser Praktiken und damit auch der Kompilationsnetzwerke betrachtet werden.

Teil dieser Praktiken waren bei Kompilationen das Sammeln von Wissensbestandteilen, die zusammengetragen wurden, um daraus neue Bücher herzustellen. Dabei wurden antike Bestände mit zeitgenössischen kombiniert, es wurde Wissen aus unterschiedlichen räumlichen und disziplinären Kontexten vermischt und neu geordnet. ${ }^{212}$ Dadurch wurde es laut Susanne Kaup auch für jene, „denen Intellekt, Zeit oder Gelegenheit fehlte, mit den Originalquellen zu arbeiten“ einfacher, Originalquellen zu studieren. ${ }^{213} \mathrm{Zu}$ diesem einfacheren Zugang habe weiter beigetragen, dass immer mehr Elemente in die Kompilationen integriert wurden -

208 Woodmansee: The Author, Art, and the Market, 1994, S. 36.

209 Ebd., S. 37.

210 Sulzer, Johann Gregor: Allgemeine Theorie der Schönen Künste in einzeln, nach alphabetischer Ordnung der Kunstwörter auf einander folgenden, Artikeln abgehandelt. Frankfurt und Leipzig, 1798.

211 Diese Vorstellung ist Teil jener des Kompilationsnetzwerks, das in Kapitel 3 konzeptualisiert wird.

212 Zum Zusammenbringen von Alt und Neu in der Frühen Neuzeit siehe Park, Katharine; Daston, Lorraine: Introduction. The Age of the New, in: Daston, Lorraine; Park, Katharine (Hg.): The Cambridge History of Science. Cambridge, UK, New York: Cambridge University Press, 2006, S. $1-17$.

213 Kaup: De beatitudinibus, 2012, S. 135. 
wie etwa Inhaltsverzeichnisse oder Indexe -, die dazu dienen sollten, Informationen rascher zu finden, die aber auch ein Verkaufsargument darstellen konnten. ${ }^{214}$ Für Kaup waren die Inhalte der exzerpierten Quellen ,geistiges Eigentum des Autors, der das Recht am und die Verantwortung für den Inhalt trägt. “215 Hier kommt ein wichtiger Punkt zum Tragen: die Verknüpfung des Autors oder der Autorin mit dem geistigen Eigentum. Sie ist ab dem 18. Jahrhundert zu beobachten und findet sich etwa bei Immanuel Kant in seiner Metaphysik der Sitten. Darin schrieb er, dass der Verleger, also jener, „,welcher durch eine Schrift im Nahmen eines Anderen (des Autors) O̊ffentlich redet“, letzteres nur dann rechtmässig täte, wenn er die Erlaubnis des Schriftstellers dafür habe. Aus diesem Grund sei auch der Nachdruck nur dann zulässig, wenn der Autor die Vollmacht gebe. ${ }^{216}$ Hier wird davon ausgegangen, dass eine Person - der Autor, die Autorin - einen Text verfasst und diesen publiziert hat. Sie ist Eigentümerin dieses Textes, der nur mit ihrer Zustimmung veröffentlicht werden darf.

Einen Einfluss auf das neue Verständnis von Autorschaft hatten - dies sicherlich auch umgekehrt - auch neue rechtliche Regulatorien, die im 18. Jahrhundert aufgrund des immer grösser werdenden Buchmarktes eingeführt wurden. ${ }^{217} \mathrm{Zu}$ diesen zählt insbesondere die Statute of Anne (8 Anne c.19), die 1710 verabschiedet wurde und die eine Rechtsgrundlage für Copyright-Vergehen bildete. ${ }^{218}$ Sie gewährte den Autorinnen und Autoren Copyright-Schutz über vierzehn Jahre und weitere vierzehn Jahre, falls der Autor oder die Autorin zu diesem

214 Ebd., S. 134; Duroselle-Melish, Caroline: Centre and Periphery? Relations between Frankfurt and Bologna in the Transnational Book Trade of the 1600s, in: McLean, Matthew; Barker, Sara (Hg.): International Exchange in the Early Modern Book World (= The Handpress World). Leiden, Boston: Brill, 2016, 31-58, hier S. 44. Siehe dazu auch: Gierl: Kompilation und die Produktion von Wissen im 18. Jahrhundert, 2001, S. 67.

215 Kaup: De beatitudinibus, 2012, S. 136.

216 Kant, Immanuel: Metaphysische Anfangsgründe der Rechtslehre. Königsberg: Friedrich Nicolovius, 1797, S. 127 f.; Johns, Adrian: Piracy. The Intellectual Property Wars from Gutenberg to Gates. Chicago: University of Chicago Press, 2009, S, S. 15; Love: Attributing Authorship, 2002, S. 41.

217 Woodmansee: The Author, Art, and the Market, 1994, S. 45; Rose: Authors and Owners, 20023, S. 6. Zum Wachstum des Buchmarktes siehe auch Vogel, Martin: Der literarische Markt und die Entstehung des Verlags- und Urheberrechts bis zum Jahre 1800, in: Rhetorik, Ästhetik, Ideologie. Aspekte einer kritischen Kulturwissenschaft. Stuttgart: J. B. Metzlersche Verlagsbuchhandlung, 1993, S. 117-136, hier S. 125.

218 Woodmansee: The Author, Art, and the Market, 1994, S. 45; Schellenberg, Betty A.: The Eighteenth Century Print, Professionalization, and Defining the Author, in: Berensmeyer, Ingo; Buelens, Gert; Demoor, Marysa (Hg.): The Cambridge Handbook of Literary Authorship. Cambridge: Cambridge University Press, 2019, S. 133-146, hier S. 139.f.; Hirschi: Compiler into Genius, 2013, S. 155 f. Zu vorherigen Regulatorien siehe Johns: The Nature of the Book, S. 232 
Zeitpunkt noch lebte. ${ }^{219}$ Ähnliche Regulatorien wurden auch in anderen Ländern eingeführt, so etwa 1790 in den Vereinigten Staaten sowie 1793 in Frankreich. ${ }^{220}$ Diese neuen Privilegien unterscheiden sich von früheren Privilegien dadurch, dass sie nicht mehr auf den Verleger oder die Verlegerin, sondern auf die Autorin oder den Autoren ausgelegt waren. ${ }^{221}$ Damit geht es auch um die Frage des Eigentums: es geht von der Verlegerin respektive dem Verleger zu Autorin respektive zum Autor über. Diese Verbindung macht nach Mark Rose den modernen Autor und wohl auch die Autorin - aus: „The distinguishing characteristic of the modern author, I propose, is proprietorship; the author is conceived as the originator and therefore the owner of a special kind of commodity, the work." ${ }^{222}$ Hier wird Autorschaft klar mit Eigentum verknüpft, mit Eigentum am eigenen Werk. Diese Vorstellung ist für das 16. Jahrhundert, auf dem der Schwerpunkt dieses Buches liegt, nicht vorhanden. ${ }^{223}$ Bei Wecker scheint das Eigentum materiell gewesen zu sein: Das Manuskript als Gut wurde dem Drucker oder der Druckerin respektive dem Verleger oder der Verlegerin verkauft. Damit ging es um die Proprietät eines Manuskripts oder Buches - und nicht um den in diesem gespeicherten Text. Dabei ist Originalität kein Thema, im Gegensatz zum genieästhetischen Autorbegriff, der vom „Original-Genie“ ausgeht. 224 Dieses Autorverständnis unterscheidet sich

219 Schellenberg: The Eighteenth Century Print, Professionalization, and Defining the Author, S. 140 .

220 Atkinson, Benedict; Fitzgerald, Brian: A Short History of Copyright. The Genie of Information. Cham [etc.]: Springer International Publishing, 2014, S. 3.

221 Vgl. Chartier, Roger: The Order of Books. Readers, Authors, and Libraries in Europe between the Fourteenth and Eighteenth Centuries. Stanford, Calif.: Stanford University Press, 1994, S. 32; Woodmansee: The Author, Art, and the Market, 1994, S. 45. Zu früheren Privilegien siehe u.a. Vogel: Der literarische Markt und die Entstehung des Verlags- und Urheberrechts, 1993, insbes. S. 119; Armstrong, Elizabeth: Before Copyright. The French book-privilege System 1498-1526 (= Cambridge Studies in Publishing and Printing History). Cambridge: Cambridge University Press, 2002.

222 Rose: Authors and Owners, 2002, S. 1.

223 Eine solche Vorstellung kann für das 16. Jahrhundert wie für das Mittelalter nicht angenommen werden. So schreibt Horst Wenzel: „Was wir unter dem modernen Begriff des Autors fassen, hat kein Äquivalent im Mittelalter.“ Wenzel, Horst: Autorenbilder. Zur Ausdifferenzierung von Autorfunktionen in mittelalterlichen Miniaturen, in: Andersen, Elizabeth; Haustein, Jens; Simon, Anne u. a. (Hg.): Autor und Autorschaft im Mittelalter. Kolloquium Meißen 1995. Tübingen: De Gruyter, 1998, S. 1-28, hier S. 11.

224 Begemann: Der Körper des Autors, 2002, S. 46. Eine solche Vorstellung kann für das 16. Jahrhundert wie für das Mittelalter nicht angenommen werden. So schreibt Horst Wenzel: „Was wir unter dem modernen Begriff des Autors fassen, hat kein Äquivalent im Mittelalter.“ Wenzel, Horst: Autorenbilder. Zur Ausdifferenzierung von Autorfunktionen in mittelalterlichen Miniaturen, in: Andersen, Elizabeth; Haustein, Jens; Simon, Anne u.a. (Hg.): Autor und Autorschaft im Mittelalter. Kolloquium Meißen 1995. Tübingen: De Gruyter, 1998, S. 1-28, hier S. 11. 
nach Christian Begemann vom vorherigen dadurch, dass er sich nicht mehr an Normen orientierte, sondern dass er „sein eigenes Sein sui generis“ darstellte. ${ }^{225}$ Hier kristallisiert sich ein wichtiger Punkt heraus: Die Vorstellung des „geistigen Eigentums“ ist geknüpft an jene des Autors als kreativer, „genialer“ Urheber eines Werkes. ${ }^{226}$ Und letzterer wiederum ist jener, der plagiiert werden kann. Dies erklärt auch, weshalb Adrian Johns die Entstehung der Vorstellung des ,geistigen Eigentums“ auf das 19. Jahrhundert datiert: Vorbedingung dafür war die Entstehung des genieästhetischen Autorbegriffs. ${ }^{227}$

Doch auch vor dem 19. Jahrhundert gab es eine Vorstellung von Plagiat, wenn auch ohne die rechtsgültige Verknüpfung von Autor respektive Autorin und „geistiges Eigentum“ - dies schon seit der Antike. Dieser Plagiatsbegriff steht für eine irreguläre Verwendung von Literatur. ${ }^{228}$ Man konnte sich etwa in der Frühen Neuzeit Plagiatsvorwürfen ausgesetzt sehen. Dies geschah zum Beispiel Walther Hermann Ryff (nach 1500 - 1548), der unter anderem Bücher in der Pharmazie, der Medizin, der Botanik, der Architektur und der Mathematik publizierte. ${ }^{229}$ Ryff wiederum wurde laut Andrea Carlino durch Cornelis Bos (1506/10 - 1555) plagi-

225 Begemann: Der Körper des Autors, 2002, S. 46. Hinzuzufügen ist, dass Einteilung in vorher und nachher simplifizierend ist und dass vorher weder alles reglementiert noch nachher alles frei war. Siehe dazu ebd., S. 47.

226 Vgl. Hartling, Florian: Der digitale Autor. Autorschaft im Zeitalter des Internets, Teilw. zugl.: Halle, Univ., Philosophische Fakultät II, Diss., 2007 u.d.T.: Hartling, Florian: Der digitale Autor? Zur Autorenschaft unter den Bedingungen des Dispositivs Internet (= Kultur- und Medientheorie). Bielefeld: Transcript-Verlag, 2009, S. 77. In diesem Zusammenhang wird auch vom „Original-Genie“ gesprochen. Begemann: Der Körper des Autors, 2002, S. 46.

227 Johns, Adrian: Piracy. The Intellectual Property Wars from Gutenberg to Gates. Chicago: University of Chicago Press, 2009, S, S. 15. Siehe auch Love, Harold: Attributing Authorship. An Introduction. New York: Cambridge University Press, 2002, S. 41. Die genieästhetische Autorschaftsvorstellung wurde insbesonders in den 1960er Jahren stark kritisiert. Siehe dazu Fussnote 121.

228 Biørnstad, Hall: Introduction, in: Biørnstad, Hall (Hg.): Borrowed Feathers: Plagiarism and the Limits of Imitation in Early Modern Europe. Oslo: Oslo Academic Press, 2008, S. 5-17, hier S. 5; Cook: Plagiarism and Proprietary Authorship in Early Modern England, 2011, S. 5; Hirschi, Caspar: Piraten der Gelehrtenrepublik. Die Norm des sachlichen Streits und ihre polemische Funktion, in: Bremer, Kai; Spoerhase, Carlos (Hg.): Gelehrte Polemik. Intellektuelle Konfliktverschärfungen um 1700. Heft 2/3 (= Zeitsprünge). Frankfurt am Main: Vittorio Klostermann, 2011, S. 176-214.

229 So etwa von Leonhard Fuchs (1501-1566), Andreas Vesalius (1514-1564) und Conrad Gessner. Kranich-Hofbauer: Walther Hermann Ryff, 2012, S. 89; Carlino: Kunstbüchlein and Imagines Contrafactae, 2008, S. 89-91; Keil, Gundolf: Ryff, Walther, in: Neue Deutsche Biographie 22, 2005, S. 310f. Online unter: https://www.deutsche-biographie.de/pnd118604376.html [25.02.2021]. 
iert. ${ }^{230}$ Plagiatsvorwürfe scheinen auch Wecker betroffen $\mathrm{zu}$ haben, nicht für seine Books of Secrets, sondern für die Medicae utriusque syntaxes, weshalb er sich 1573 entschied, dem vorzugreifen: „Alleß waß Mathiolus [Petrus Andreas Matthiolus (1501-1577), SZ] für sein eigen gehalten, hab ich hin weg gethon, vnd geendert“, schrieb er an Theodor Zwinger. ${ }^{231}$ Solche Vorwürfe konnten folglich zu einem veränderten Umgang anderer Kompilierender mit einem Text führen. Sie konnten aber auch ökonomisch wertvoll sein: Sie waren ein Mittel, den eigenen Namen ins Gespräch und jenen des Kollegen oder der Kollegin in Verruf zu bringen, was den eigenen Erfolg befördern konnte. ${ }^{232}$ Das Plagiat war im 16. Jahrhundert demnach nicht an eine rechtliche Instanz gebunden, konnte aber dennoch Auswirkungen auf den Text haben. Damit konnten Plagiatsvorwürfe als äussere Faktoren auf den am Ende produzierten Text einwirken.

Für Matíaz Martínez stellt das Plagiat „eine besonders intensive, andererseits aber eine defizitäre Form von Intertextualität“ dar, weil es den intertextuellen Bezug vor den Leserinnen und Lesern verberge. ${ }^{233}$ Laut William Eamon habe man in der Frühen Neuzeit jedoch auch dann als Plagiator bezeichnet werden können, wenn man den Bezug kenntlich gemacht habe. ${ }^{234}$ Damit unterscheidet sich der frühneuzeitliche Plagiatsbegriff auch in diesem Punkt von dem modernen: Er war weiter gefasst und konnte auch Text- und Bildübernahmen miteinschliessen, die heute als Zitate und nicht als Plagiate betrachtet werden würden. Zudem darf folgender Aspekt nicht vernachlässigt werden, auf den Eamon aufmerksam macht: „Almost every author ,borrowed“ material from others, with or without acknowledgement, and thus, most inevitably, risked being called a plagiarist. “235 Diese Aussage betont die Alltäglichkeit des Übernehmens von bestehendem Material: Plagiarismus war Teil der frühneuzeitlichen Textproduktionspraktik der

230 Carlino: Kunstbüchlein and Imagines Contrafactae, 2008, S. 91. Zu Walther Hermann Ryff und seinem „Plagiarismus“ siehe auch Marr, Alexander: Walther Ryff, Plagiarism and Imitation in Sixteenth-Century Germany, in: Print Quarterly 31 (2), 2014, S. 131-143.

231 Wecker, Johann Jacob: Brief an Theodor Zwinger. UB Basel, Frey-Gryn Mscr II 28:Nr. 371. Dieser Plagiatsvorwurf findet sich bei Thomasius, Jacobus; Reinelius, Johannes Michael: Dissertatio philosophica de plagio literario [...]. Buchta: Levcopetræ literis Joh. Brühlii, 1679, S. 30 f. Siehe dazu auch [Anonym]: Art. „Wecker, (Johann Jacob)“, in: Zedler, Universallexicon, Sp. 1772. 232 Siehe dazu auch Biørnstad: Introduction, 2008, S. 7.

233 Martínez: Autorschaft und Intertextualität, 2000, S. 469. Eine negative Bewertung findet sich auch bei anderen Plagiatsbegriffen, wie etwa jenem Harold Loves: „In cases where an author makes extensive unacknowledged use of the words of other authors we have what is now called plagiarism (still a form of authorship, albeit a discreditable one).“ Love: Attributing Authorship, 2002, S. 40.

234 Eamon: Science and the Secrets of Nature, 1996, S. 96.

235 Ebd. 
Kompilation und damit Teil der wohl wichtigsten Textproduktionspraktiken der Zeit. $^{236}$

Doch wer war alles involviert in diese Plagiate oder Textübernahmen? Welches Autorschaftsverständnis ist anwendbar auf die Praktik der Kompilation in der Frühen Neuzeit? Aufgrund der Tatsache, dass Bücher zumeist einer Person zugeschrieben werden, könnte man davon ausgehen, dass das Autorschaftskonzept im Sinne einer Person, die hinter einem Text steht, auch auf Kompilationen übertragen werden kann. Das Fallbeispiel Johann Jacob Weckers zeigt jedoch, dass dies zu simplifizierend ist: Hinter einer Kompilation stand nicht eine einzelne Person, sondern ein Netzwerk bestehend aus mehreren Akteurinnen und Akteuren sowie Dingen und äusseren Faktoren - ein Kompilationsnetzwerk. Die Vorstellung des Kompilationsnetzwerks baut auf bestehenden Konzepten der kollaborativen Autorschaft auf, wobei hier im Besonderen auf jenes Harold Loves eingegangen wird, da viele Elemente davon auf die Frühe Neuzeit übertragen werden können, auch wenn sein Konzept für die Moderne entwickelt wurde. ${ }^{237}$

\subsection{Kollaborative Formen von Autorschaft}

Der Lovesche Autorschaftsbegriff ist ein kollaborativer und wie folgt definiert:

The term 'authorship' [...] [does, SZ] not therefore denote the condition of being an originator of works, but a set of linked activities (authemes) which are sometimes performed by a single person but will often be performed collaboratively or by several persons in succession. ${ }^{238}$

Damit betont er den häufig kollaborativen Charakter von Texten, wie dies auch in den Literaturwissenschaften immer öfters konstatiert wird. ${ }^{239}$ Demnach geht er nicht von einer Person als Urheber oder Urheberin eines Werks aus. ${ }^{240}$ Die Begriffe „collaboration“ und „collaborative authorship“ werden nach Heather Hirschfeld für diverse Interaktionen im Zusammenhang von ,shaping the meaning and significance of a text“ verwendet. ${ }^{241}$ Dazu zählten das gemeinsame Schreiben eines Textes sowie die enge Zusammenarbeit mit Druckenden und Lesenden. ${ }^{242}$

236 Vgl. ebd.; Hertel: Kompilieren, Klassifizieren, Contrafactieren, 2008, S. 4.

237 Love: Attributing Authorship, 2002, u. a. S. 7. Auch andere Autoren haben für eine „Rückkehr des Autors“ plädiert, u.a. Burke: The Death and Return of the Author, $1999^{2}$.

238 Love: Attributing Authorship, 2002, S. 39.

239 Vgl. Hirschfeld: Early Modern Collaboration and Theories of Authorship, 2001, S. 610.

240 Love: Attributing Authorship, 2002, S. 39. Siehe auch ebd., S. 7.

241 Hirschfeld: Early Modern Collaboration and Theories of Authorship, 2001, S. 610. Auch Jefferey Masten betont die grosse Bedeutung der Kollaboration für die Textproduktion des 16. und 
Die Love’sche Vorstellung einer „kollaborativen Autorschaft“ umfasst hingegen nicht nur die Zusammenarbeit rund um das Schreiben und Drucken, sondern auch den Spracherwerb, die Ausbildung, Schreiberfahrungen, Gespräche, das Lesen von anderen Autoren sowie alle Schritte nach dem Verfassen des Textes, wie etwa das Einarbeiten von Korrekturvorschlägen von Gegenleserinnen und Gegenlesern sowie die Bearbeitung des Textes für den Druck. ${ }^{243}$ Wie die darin involvierten Personen miteinander agieren, beschreibt Love in vier Kategorien von Autorschaft.

Die erste ist jene der „precursory authorship“, die dann stattfindet, wenn eine kollaborative Autorschaft mit einem „signifikanten Beitrag“ eines früheren Autors oder einer Autorin arbeitet. ${ }^{244}$ Was unter einem „signifikanten Beitrag“ verstanden wird, bleibt dabei unklar. In jedem Fall wird jedoch der oder die Verfassende dieses Beitrags zum Teil der Autorschaft. ${ }^{245}$ Im Falle der Weckerschen Buchproduktion wären die „authores“, aus denen kompiliert wurde, „precursory authors“: Sie dienten als Quellen und wirkten auf den Text ein.

Als zweite Kategorie nennt Love jene der „executive authorship“, die sich auf den Kompilatoren oder die Kompilatorin des „verbal texts“ bezieht bis zu jenem Zeitpunkt, bei dem der Text als publikationsfähig betrachtet wird. ${ }^{246}$ Diese Art der Autorschaft könne, müsse aber nicht kollaborativ sein. ${ }^{247}$ Man könnte sagen, dass Johann Jacob Wecker und wohl auch Theodor Zwinger die exekutiven Autoren vieler Weckerschen Publikationen waren. Sie verarbeiteten das Material so, dass der Text publikationsfähig wurde. Auch Übersetzerinnen und Übersetzer sowie Ghostwriterinnen und Ghostwriter können „executive authors“ sein. Sie leisten viel Textarbeit, werden aber nicht zwingend als Autorinnen oder Autoren wahrgenommen. Blickt man auf die „executive authorship“, so zoomt man sozusagen nur auf einen Teil der Textproduktion und lässt die anderen beeinflussenden Faktoren ausser Acht. Diese sind jedoch ebenfalls von Bedeutung, wenn man die Urheberschaft eines Textes betrachten möchte.

des 17. Jahrhunderts. Masten, Jeffrey: Textual Intercourse: Collaboration, Authorship, and Sexualities in Renaissance Drama. Cambridge: Cambridge University Press, 1997, S. 4. Gleiches gilt für Smith, Helen: Grossly Material Things. Women and Book Production in Early Modern England. Oxford: Oxford University Press, 2012, S. 4f.; Martins: Les livres de secrets imprimés et traduits en Europe, 2015, S. 162f. sowie Duroselle-Melish: Centre and Periphery?, 2016, S. 42.

242 Hirschfeld: Early Modern Collaboration and Theories of Authorship, 2001, S. 610.

243 Love: Attributing Authorship, 2002, S. 33.

244 Ebd., S. 40.

245 Ebd.

246 Ebd., S. 43.

247 Ebd. 
Im Gegensatz zur „executive authorship“ geht es bei der dritten Kategorie, der „declarative authorship“, nicht um konkrete Textproduktion, sondern um die Zuschreibung von Text zu einer Person, die diesen gar nicht unbedingt verfasst hat. ${ }^{248}$ Ein Beispiel dafür sind Biographien berühmter Persönlichkeiten, die als Autobiographien deklariert werden, auch wenn sie nicht von der Person selbst geschrieben wurden. Wie es der Begriff impliziert, geht es bei der „declarative authorship“ um eine Funktion, die nach aussen orientiert ist:

A second function of the declarative author is that of 'owning the words' - of appearing in the public sphere as the work's creator, and of shouldering the responsibilities and accepting the benefits that flow from this: indeed, it is only through performing the declarative part of authorship that one can figure oneself as an author [...]..$^{249}$

Inwiefern es hier nur um die Repräsentation gegen aussen über den Namen auf der Titelseite geht, oder aber durch eine in personam, bleibt unklar. Beides kann der Fall sein. Klar ist aber, dass die Zuschreibungen eines Textes zu einem Autor oder einer Autorin die Rezeption beeinflussen kann. ${ }^{250}$ Es scheint mir, dass man den Namen Johann Jacob Weckers durchaus in der Funktion der deklarativen Autorschaft verwendet hat. ${ }^{251}$

Die vierte Unterkategorie bezeichnet Love als „revising authorship“. Dabei bezieht er sich auf - insbesondere stilistische - Überarbeitungen von Texten. Diese Form der Autorschaft könne nicht immer von der ursprünglichen Komposition des Textes getrennt werden, da sich beide Prozesse überlagern könnten. ${ }^{252}$ Der Überarbeitungsprozess sei durch das Schreiben am Computer komplexer geworden, weshalb nicht immer von einem linearen Prozess des Schreibens, Überarbeitens und Publizierens ausgegangen werden könne. Love sieht das Überarbeiten auch kritisch:

[...] living as we do in a culture which places great stress on individuality, and often being attracted to writers by our perception of that individuality, we have a strong predisposition to prefer the authentic and unpolished over the version perfected by an alien hand..$^{253}$

248 Ebd., S. 45.

249 Ebd.

250 Ebd., S. 46.

251 Siehe z.B. Wecker: De Secretis libri sex, 1559; Wecker: De Secretis libri XVII, 1582; Wecker, Read: Eighteen Books of the Secrets of Art \& Nature, 1660.

252 Love: Attributing Authorship, 2002, S. 46.

253 Ebd., S. 47. 
Dieses Zitat macht deutlich, dass es sich bei der Autorschaftsvorstellung Loves wie bei der traditionellen Autorschaftsvorstellung um eine individualistische handelt. „Collaboration“ bezieht sich bei Love auf die Zusammenarbeit unterschiedlicher Akteure, die jeweils ihr eigenes „Genie“, wie man es im 18. Jahrhundert wohl gesagt hätte, einbringen und so auf den Text einwirken. Damit ist ein eigener Stil oder aber einer, der von einer Gruppe gewollt wird, gemeint. Der wichtigste Punkt hier ist, dass es bei der Loveschen Autorschaftsvorstellung noch immer um die Produktion eines Textes geht, bei dem „geistiges Eigentum“ und individuelles „Genie“ präsent ist. ${ }^{254}$ Diese Verknüpfung trifft jedoch nicht bei allen Publikationen zu: Gerade bei frühneuzeitlichen Kollaborationen, wie etwa von Kompilationsnetzwerken, war die Vorstellung vom „individuellen Genie“ nicht relevant. Johann Jacob Wecker schrieb zum Beispiel an Theodor Zwinger (1533-1588): „Daß [Book of Secret, SZ] hab ich so groß gemacht, als mir müglich gwesen, het ich mher aůthores gehapt oder gewüst, welte ichs auch vil groser gemacht haben." ${ }^{255}$ Folglich ging es ihm nicht um eigene Ideen, die verbreitet werden sollten, sondern um jene bestehender „authores“. Dabei bezog er sich auch kaum auf neu Geschriebenes, das verarbeitet werden sollte, wie dies bei der „precursory authorship“ der Fall wäre, sondern auf Bücher, aus denen er kompilierte.

Im Gegensatz zu Love spielt bei der Autorschaftsvorstellung von Helen Smith die Materialität eine grosse Rolle: Für sie waren kreative Handlungen in der Buchproduktion sowohl von Netzwerken, in die Frauen und Männer involviert waren, als auch von materiellen und institutionellen Kontexten beeinflusst. ${ }^{256}$ Diese unterschiedlichen Elemente bildeten, als „grossly material things“, eine Art Spinnennetz, das textuelle Diskursstränge mit Menschen und Dingen verbunden habe, „which shape and are shaped by it“. ${ }^{257}$ Aus diesem Grund könne auch nicht von männlicher oder weiblicher Autorschaft gesprochen werden, sondern von einem Interface, auf dem mehrere Akteurinnen und Akteure auf komplexe und

254 Ähnliche Vorstellungen von kollektiver Autorschaft finden sich auch bei anderen Forschenden; sie beziehen sich häufig auf jene frühneuzeitlicher Dramen. Hirschfeld: Early Modern Collaboration and Theories of Authorship, 2001, S. 611, 620.

255 Wecker, Johann Jacob: Brief an Theodor Zwinger. UB Basel, Frey-Gryn Mscr II 4:Nr. 326.

256 Smith: Grossly Material Things, 2012, S. 6. Zur Involviertheit von Frauen im Buchdruck siehe u.a. Gardner, Victoria: Introduction: Practices, Perceptions and Connections, in: Hinks, John; Gardner, Victoria E. M. (Hg.): The Book Trade in Early Modern England. Practices, Perceptions, Connections. New Castle, London: The British Library Publishing Division, 2014, S. VII-XV, XI.; Bell: Women and the Production of Texts: the Impact of the History of the Book, 2014, S. 108, 119; MacDowell, Paula: The Women of Grub Street. Press, Politics, and Gender in the London Literary Marketplace, 1678-1730. Oxford, New York [etc.]: Clarendon Press, 1998, S. 26.

257 Smith: Grossly Material Things, 2012, S. 6. Zitat: S. 10. 
unterschiedliche Art und Weise zusammen interagierten. ${ }^{258}$ Im Gegensatz zu Smith sehe ich das Netz, in das Wecker eingebunden war, nicht als von einem Kern ausgehend, sondern multidimensionaler und relationaler. Auch ich nehme an, dass Dinge in dieses Netz eingebunden waren und Einfluss auf den Text nahmen. Hier spielt die Idee mit hinein, dass Menschen und deren soziales Leben von Dingen beeinflusst sind. ${ }^{259}$ Letztere wirken auf die Praktiken der Menschen ein, „weder ausschließlich als zu bearbeitende Objekte noch als Kräfte eines physischen Zwangs. “260 Dies gilt auch für die Buchproduktion, wie es Francis Donald McKenzie deutlich gemacht hat:

\begin{abstract}
Almost all texts of any consequence are the product of the concurrent inter-action of ideologies and institutions, of writers, publishers, printers, binders, wholesalers, travellers, retailers, as well as of the material sources (and their makers and suppliers) of type, paper, cord, and all the appurtenances of a printing house. ${ }^{261}$
\end{abstract}

All diese Elemente wirken auf die Text- und auch die Buchproduktion ein und sind deshalb alle als Teile des Kompilationsnetzwerks zu verstehen.

Die unterschiedlichen Vorstellungen von kollaborativen Autorschaften in der Forschung beziehen im Gegensatz zum traditionellen Autorbegriff mehrere Akteurinnen und Akteure in die Textproduktion mit ein und berücksichtigen auch externe Faktoren. ${ }^{262}$ Sie haben einen direkten Bezug zur Textproduktion, während das Konzept des Kompilationsnetzwerks auch Akteurinnen und Akteure sowie Elemente integriert, die nicht direkt mit den Büchern beschäftigt waren, aber etwa an der Korrespondenz und/oder Beschaffung mitwirkten. Diese Handlungen bildeten, im Gegensatz zu vielen anderen Textgattungen, die wichtigste Grundlage der Kompilationsproduktion. Denn bei Kompilationen stand das Sammeln und Neuordnen von Textbestandteilen im Vordergrund und nicht das kreative

258 Ebd., S. 4.

259 Hodder, Ian: Human-Thing Entanglement. Towards an Integrated Archeological Perspective, in: Journal of the Royal Anthropological Institute 17, 2011, S. 154-177, S. 155.

260 Hörning, Karl H.; Reuter, Julia: Doing Culture: Kultur als Praxis, in: Hörning, Karl H.; Reuter, Julia (Hg.): Doing Culture. Neue Positionen zum Verhältnis von Kultur und sozialer Praxis (= Sozialtheorie). Bielefeld: transcript, 2004 S. 9-15, hier S. 11. Hörning und Reuter verweisen auf Reckwitz: Reckwitz, Andreas: Grundelemente einer Theorie sozialer Praktiken. Eine sozialtheoretische Perspektive, in: Zeitschrift für Soziologie 32 (4), 2003, S. 282-301, hier S. 291.

261 McKenzie, Donald Francis: 5. The London Book Trade in 1644, in: McKenzie, Donald Francis; McDonald, Peter D.; Suarez, Michael F. (Hg.): Making Meaning. „Printers of the Mind“ and other Essays (= Studies in Print Culture and the History of the Book). Amherst: University of Massachusetts Press, 2002, S. $126-143$, hier S. 128.

262 Siehe auch Hirschfeld: Early Modern Collaboration and Theories of Authorship, 2001, S. 614. 
Schreiben eines neuen Textes. Dies bedeutet, dass den Büchern in ihrer Materialität eine grössere Bedeutung zugesprochen werden muss als bei anderen Textgattungen. Um solche Bücher herzustellen, wurden Textbestandteile verschiedenster Autoritäten wiederverwertet und zusammengebracht, was der Titel des folgenden Traktats verdeutlicht: Artznney Büchlein wider allerley kranckheiten vnd gebrechen der Zene gezogen aus dem Galeno Auicenna Mesue Cornelio Celso vnd anderen mehr der Artzney Doctorn sehr nuetzlich zu lesen. ${ }^{263}$ Diese Vielfältigkeit war typisch für die frühneuzeitliche Kompilation. ${ }^{264}$ Dies gilt auch für das 1570 erschienene Kunstbuoch: Deß hocherfarnen vnnd Weytberhuempten Herrn Gabrielis Fallopij. ${ }^{265}$ In der Vorrede ist $\mathrm{zu}$ lesen, dass darin Secreta von vielen trefflichen Doctores enthalten seien, „als da seind / Ioannes Essenus, des Koenigs auss Engelland / vnd Rogoricus Gariglius / dess Cardinals Paceci / vnd dessen von Guisen Leibartzet / vnnd andere vil mehr / welche zum theyl mit todt abgangen / zum theil aber noch im leben seind / als nemblich der hochberuempte Matheus Curtius, Bellocatus, Trincauella, Francazanus / vnd Victorius Fauentius. Wil geschweigen / das er vndter den eltesten Artzeten die fürnembste auch einfuehrt / als Auicennam, Serpionem, Rabbi Mosem, Auerrhoem / vnd Arnoldum de villa noua, \& c." ${ }^{266}$ Daraus sei einfach abzuleiten, dass die Inhalte dieses Buches nicht so unbedeutend seien, wie es „mancher vnuerstendiger aus dem Titel vnd vberschrifft diss Bůchs villeicht vnbedechtlicher weiss vrtheilen moechte.“267 Diese Autoritäten lassen sich in sozialer Hinsicht unterschiedlich verorten, waren Ärzte oder auch nicht, einige waren schon lange verstorben, andere noch am Leben. ${ }^{268}$ Diese Aufzählung sollte zeigen, dass es sich um „nit so geringe sachen“ handelte: Die „authores“ sollten folglich dem darauffolgenden Text mehr Gewicht geben und der Beglaubigung dienen. ${ }^{269}$ Im Text selbst finden sich jedoch keine direkten Verweise auf die in der Vorrede genannten Autoritäten. ${ }^{270}$ Die Aufzählung ver-

263 [Anonym]: Artznney Büchlein wider allerley kranckheiten vnd gebrechen der Zene gezogen aus dem Galeno Auicenna Mesue Cornelio Celso vnd anderen mehr der Artzney Doctorn sehr nuetzlich zu lesen, in: Schellenberg, Tarquinius (Hg.): Artzney buch, Köstlich fur mancherley Kranckheit des gantzen leibs, Jnnerlich vnd eusserlich, vom Heupt an, bis auff die Füsse. Erfurrdt: Welther Sathssen, 1546, hier [S. 316].

264 Siehe Einleitung, Kapitel 1.

265 Falloppio, Gabriele: Kunstbůch: Deß hocherfarnen vnnd Weytberhůmpten Herrn Gabrielis Fallopij, der Artzney Doctorn von mancherley nutzlichen bißher verborgnen vnd lustigen Ko̊nsten. [s.l.]: [s.n.], 1570.

266 Ebd., fol. 5 f.

267 Ebd.

268 Ebd.

269 Ebd.

270 Ebd. 
deutlicht aber, dass sowohl die Vielzahl als auch die Vielfalt an Autoritäten und deren Bücher bedeutsam für die Anpreisung eines Buches war.

Im Gegensatz zum Kunstbuch des Falloppius listet Wecker die von ihm verwendeten ,authores“ in vielen Publikationen auf. Er steigert die Anzahl der Autoritäten im Vergleich zu den vorher betrachteten Publikationen um ein Mehrfaches: In den De Secretis libri XVII von 1582 sind 129 Autoritäten aufgeführt, aus denen kompiliert wurde. Diese Liste ist jedoch nicht vollständig: Im Text selbst führt Wecker weitere „authores“ von Textbestandteilen aus Büchern auf. ${ }^{271}$ Auch das 1616 publizierte des Kunstbuch Des Wolerfarnen Herren Alexii Pedemontani wird auf viele verschiedene Autoritäten abgestützt, wie die auf das Jahr 1571 datierte Vorrede Weckers verdeutlicht:

Es haben nicht nur die berühmtesten und höchsten Ärzte Galen, Avicenna, Aegineta, Aetius [...] viele herzliche, vortreffliche und bewährte Arzneimittel für die Gesundheit und die Verschönerung und Wohlgestalt des Leibes beschrieben, sondern auch viele der neuen Ärzte, unter welchen wir den vornehmen und wohlgelehrten Herrn Alessio Piemontese nicht als den Geringsten schätzen sollten. ${ }^{272}$

Damit werden, wie im anonym erschienenen Artznney Büchlein, antike Autoritäten erwähnt, im Gegensatz zu letzterem jedoch auch neue, also zeitgenössische, Ärzte. ${ }^{273}$ Auf diese Weise reihte sich Wecker und sein Kompilationsnetzwerk in die frühneuzeitliche Kompilationspraktik ein, die neue und alte Autoritäten miteinander verband. ${ }^{274}$ Dies kann auch für die De Secretis aus dem Jahr 1582 festgestellt werden, in denen unter anderem Aristoteles, Cleopatra, Cornelius Agrippa, Felix Platter, Galen, Conrad Gessner sowie Hippocrates erwähnt werden. ${ }^{275}$ Wie diese Auswahl zustande kam, ist schwierig zu rekonstruieren. Ein wichtiges Ziel war jedoch, so viele „authores“ wie möglich zu versammeln. Dabei ging es einerseits um die erwähnte Beglaubigung des Buches. Andererseits lag dies auch darin begründet, dass der Ausgabe von 1582 ein gewisser Universalitätsanspruch in-

271 Siehe Wecker: De Secretis libri XVII, 1582, passim.

272 „Es haben nicht allein die beruemptesten vnd hoechsten Aertzet Galenus Avicenna Egineta Aetius [...] viel herzliche treffenliche vnnd bewerte Artzneyen zu gesundtheit vnd zu der zierung oder wolgestaltung des Leibes beschrieben sonder auch vil der newen Aertzet unter welchen wir den fuernemmen vnnd wolgelerten Herren Alexium Pedemontanum nicht fuer den geringsten schetzen sollen." Wecker, Johann Jacob: Kunstbuch Des Wolerfarnen Herren Alexii Pedemontani von mancherleyen nutzlichen unnd bewerten Secreten oder Künsten Der Ander Theil. [...]. Basel: König, 1616.

273 [Anonym]: Artznney Büchlein wider allerley kranckheiten [...], 1546. Zitat: Ebd., [s.p.].

274 Vgl. Kaup: De beatitudinibus, 2012, S. 135.

275 Wecker, Johann Jacob: De Secretis libri XVII. Ex varijs authoribus collecti, methodice'que digesti. Basileae: [s.typ.], 1582. Augsburg, Staats- und Stadtbibliothek, Med 4773. 
härent ist. Denn nur vielfältiges Wissen konnte all die Bereiche abdecken, die Wecker in dieser Publikation thematisierte. Dazu gehörten nicht nur die bereits in den Piemonteseschen Publikationen aufzufindenden Rezepte zur Herstellung von Produkten wie Seifen, destillierten Wassern oder Nahrungsmitteln, sondern auch Informationen zu Gott und den Engeln, zu den artes liberales, zu den artes mechanices, zur Magia naturalis oder zu verschiedenen Naturgewalten. ${ }^{276}$ All diese Wissensbestände zu sammeln, sie in eine neue Ordnung zu bringen und dadurch neues Wissen zu generieren, wäre für eine Person allein nicht möglich gewesen. Denn in der Frühen Neuzeit mussten Bücher zum Herauskopieren in ihrer Materialität beschafft und dazu transferiert werden. Aus diesem Grund war für diese Art der Textproduktion das Kompilationsnetzwerk unabdingbar.

Kompilationsnetzwerke finden sich nicht nur bei Books of Secrets, sondern auch bei anderen Rezeptbüchern. Doch was sind überhaupt Books of Secrets und Rezeptbücher und inwiefern unterscheiden sie sich voneinander? Auf diese Frage und die entsprechende Forschung wird im folgenden Unterkapitel eingegangen, um deutlich zu machen, welche Art von Text in dieser Arbeit untersucht wird.

\subsection{Rezeptbücher und Books of Secrets}

Rezeptbücher beinhalten Anleitungen zur Herstellung von Produkten. Dies gilt für Koch-, Distillier-, aber auch medizinische Rezeptbücher. ${ }^{277}$ Pamela H. Smith bezeichnet diese Texte als „technical writing“, andere Forschende sprechen von „how-to“-Büchern. ${ }^{278}$ Der praktische Aspekt dieser Texte ist nach Pamela H. Smith in Books of Secrets auch in den Übersetzungen ersichtlich. ${ }^{279}$ Dabei bezieht sie sich auf die Weckersche Übersetzung der Piemonteseschen Secreti als Kunstbuch de $\beta$ wolerfaren Herren Alexij Pedemontani von mancherley nutzlichen unnd be-

\section{Ebd.}

277 Vgl. Smith: What is a Secret?, 2011, S. 52.

278 Ebd., S 53. Ebenso Tebeaux, Elizabeth: Women and Technical Writing, 1475-1700: Technology, Literacy and Developement of a Genre, in: Hunter, Lynette; Hutton Sarah (Hg.): Women, Science and Medicine, 1500 -1700. Mothers and Sisters of the Royal Society. Stroud: Sutton Pub., 1997, S. 29 - 62. Von „how-to“-Büchern sprechen u. a. Smith: What is a Secret?, 2011, S. 54; Cabré, Montserrat: Keeping Beauty Secrets in Early Modern Iberia, in: Leong, Elaine; Rankin, Alisha Michelle (Hg.): Secrets and Knowledge in Medicine and Science, 1500-1800 (= The History of Medicine in Context). Farnham: Routledge, 2011, S. 167-190, hier S. 168; Carlino: Kunstbüchlein and Imagines Contrafactae, 2008, S. 93; Gruman Martins: Os livros de segredos italianos e o desenvolvimento da ciência moderna, 2014, S. 223.

279 Smith: What is a Secret?, 2011, S. 53. 
werten Secreten oder Künsten [...]. ${ }^{280}$ Mit dieser Übertragung war Wecker nicht allein: Auch die deutsche Ausgabe der Secreti des Falloppio ist als Kunstbuch betitelt. ${ }^{281}$ Das Geheimnis war bei diesen Übersetzungen stark mit dem Begriff des Könnens verknüpft, was auch den Terminus des „how-to“-Buches erklärt. ${ }^{282}$ Letzterer kann sowohl für Books of Secrets als auch für Rezeptbücher verwendet werden.

Rezeptbücher respektive das Rezept bezeichnet Gianna Pomata als „primary epistemic genre“. Dieses umfasse Jahrhunderte der Geschichte, habe eine äusserst stabile Struktur und sei in unterschiedlichen Kulturen zu finden. ${ }^{283}$ Unter einem „epistemischen Genre“ versteht Pomata Texte, die sich parallel zur wissenschaftlichen Praxis entwickeln. ${ }^{284}$ Dies gilt meines Erachtens aber nicht nur für die einzelnen Rezepte, sondern auch für das Rezeptbuch. Nach Pomata entwickelte sich das Genre des Rezeptes zwischen dem späten Mittelalter und der Frühen Neuzeit weiter; es habe sich in formula und recipe aufgeteilt. Formula sei die theoretische und universelle Form, das recipe die individualisierte und praktische gewesen. ${ }^{285}$ Durch diese Trennung seien formulae in antidotaria und recipes in experimenta aufgeteilt worden. ${ }^{286}$ Diese Aufteilung findet sich auch im Antidotarium speciale Johann Jacob Weckers, das 1574 herausgegeben wurde. Während im ersten Teil die Grundsubstanzen beschrieben sind - quasi die Zutaten -, widmet sich der zweite Teil der Herstellung der Produkte. ${ }^{287}$ Die Books of Secrets

280 Ebd. Siehe auch Martins: Les livres de secrets imprimés et traduits en Europe, 2015, S. 148. Wecker: Kunstbuch deß wolerfaren Herren Alexij Pedemontani, 1569. Ein weiteres Beispiel ist: Wecker: Kunstbůch Des Wolerfarnen Herren Alexij Pedemontani, 1570.

281 Falloppio: Kunstbůch: Deß hocherfarnen vnnd Weytberhůmpten Herrn Gabrielis Fallopij, der Artzney Doctorn von mancherley nutzlichen bißher verborgnen vnd lustigen Könsten, 1570. 282 Siehe u. a. Smith: What is a Secret?, 2011, S. 54; Cabré: Keeping Beauty Secrets in Early Modern Iberia, 2011, S. 168; Carlino: Kunstbüchlein and Imagines Contrafactae, 2008.

283 Pomata, Gianna: The Recipe and the Case. Epistemic Genres and the Dynamic of Cognitive Practices, in: Greyerz, Kaspar von; Flubacher, Silvia; Senn, Philipp (Hg.): Wissenschaftsgeschichte und Geschichte des Wissens im Dialog - Connecting Science and Knowledge. Schauplätze der Forschung - Scenes of Research. Göttingen: V\&R Unipress, 2013, S.131-154, hier S. 136. 284 Pomata, Gianna: The Medical Case Narrative: Distant Reading of an Epistemic Genre, in: Literature and Medicine 32 (1), 2014, S. 1-23, hier S. 2. Joachim Telle spricht davon, dass das Rezept auch als „Grundform wissenschaftlicher Prosa“ bezeichnet wird. Ders.: Das Rezept als literarische Form. Zum multifunktionalen Gebrauch des Rezepts in der deutschen Literatur, in: Berichte zur Wissenschafts-Geschichte 26 (4), 2003, S. 251-274, hier S. 251.

285 Pomata: The Recipe and the Case, 2013, S. $138 \mathrm{f}$.

286 Ebd. Zum Pairing von Theorie und Praxis siehe u. a. Dear: What Is the History of Science the History Of?, 2005, S. 393.

287 Wecker: Antidotarium speciale, 1574. 
sieht Pomata als Nachfolger der experimenta. ${ }^{288}$ Dies kann für die Weckersche Buchproduktion bestätigt werden. Hinzuzufügen ist, dass die Rezeptteile der Antidotarien und jene der Books of Secrets von der Struktur her, zum Teil aber auch inhaltlich, grosse Ähnlichkeiten aufweisen.

Handschriftliche Rezeptsammlungen bezeichne ich ebenfalls als Rezeptbücher; da beide einen kompilatorischen Charakter aufweisen, standen zum Teil auch die gleichen Textproduktionspraktiken dahinter. ${ }^{289}$ Sowohl für die Herstellung von Manuskripten als auch von Drucken war zudem der Austausch im Netzwerk von grosser Bedeutung. ${ }^{290}$ Personen unterschiedlicher Schichten konnten darin involviert sein. ${ }^{291}$ Gedruckte und handschriftliche Rezeptsammlungen lagen in der Frühen Neuzeit parallel vor, wobei ein Transfer von Wissensbeständen auf beiden Seiten stattgefunden hat. ${ }^{292}$ So finden sich auch in den Weckerschen De Secretis libri XVII von 1582 Rezepte aus handschriftlichen Quellen - zumindest wird dies so deklariert. ${ }^{293}$ Ein Unterschied zwischen Druck und Manuskript liegt neben jenem der Materialität darin, dass handschriftliche Rezepte öfters mit anderen Textformen vermischt wurden, wie etwa in Briefen, Hausund Familienbüchern. ${ }^{294}$

Rezeptbücher, insbesondere handschriftliche, gelangten in den letzten Jahren vermehrt in den Blick der Forschung. Dies hängt damit zusammen, dass sich der enge Blick der Wissenschaftsgeschichte im Rahmen der kulturhistorischen Perspektivierung der 1980er Jahren geöffnet hat, wodurch auch Wissensbestände, die nicht auf den ersten Blick als ,wissenschaftliches Wissen“ betrachtet werden, in diesem Feld einen Platz gefunden haben. ${ }^{295} \mathrm{Zu}$ Rezeptbüchern als Quellen der

288 Pomata: The Recipe and the Case, 2013, S. 138 f.

289 Siehe auch Leong: Recipes and Everyday Knowledge, 2018, S. 170.

290 Rankin, Alisha Michelle: Panaceia's Daughters. Noblewomen as Healers in Early Modern Germany (= Synthesis). Chicago: University of Chicago Press, 2013.

291 DiMeo, Michelle: Authorship and Medical Networks: Reading Attributions in Early Modern Manuscript Recipe Books, in: DiMeo, Michelle; Pennell, Sara (Hg.): Reading and Writing Recipe Books, 1550 -1800. Manchester: Manchester University Press, 2013, S. 25-46, hier S. 33.

292 Siehe Adams, Thomas R.; Barker, Nicolas: A New Model for the Study of the Book, in: Barker, Nicolas (Hg.): A Potencie of Life. Books in Society (= The Clark Lectures). London: British Library, 1993, S. 5- 43, hier S. 12; Bland: Early Printed Books and Manuscripts, 2010, S. 13; Leong: Recipes and Everyday Knowledge, 2018, S.165-167, 170.

293 Wecker: De Secretis libri XVII, 1582.

294 Leong, Elaine: Collecting Knowledge for the Family: Recipes, Gender and Practical Knowledge in the Early Modern English Household, in: Centaurus 55 (2), 2013, S. 81-103; Leong: Recipes and Everyday Knowledge, 2018, u. a. S. 55, 60, 127.

295 Zedelmaier: Werkstätten des Wissens zwischen Renaissance und Aufklärung, 2015, S. 2; Brendecke, Arndt; Friedrich, Markus; Friedrich, Susanne (Hg.): Information in der Frühen Neuzeit. Status, Bestände, Strategien (= Pluralisierung \& Autorität, Bd. 16). Berlin, Münster: LIT, 2008; 
Wissensgeschichte forschen insbesondere Frauen aus dem englischsprachigen Raum, wobei der Genderaspekt eine bedeutende Rolle spielt. ${ }^{296}$ Gefragt wurde u.a. nach der Herstellung von medizinischen Produkten im Haushalt sowie nach dem Austausch von Rezepten zwischen adeligen Frauen, vorwiegend anhand von Quellen aus dem englischsprachigen Raum des 17. und 18. Jahrhunderts. ${ }^{297}$ Monica Green argumentierte in diesem Kontext, dass das Sammeln und Publizieren medizinischer Rezepte in der Frühen Neuzeit „distinctly feminine“ gewesen sei. ${ }^{298}$ Dies bezweifelt Alisha Rankin, wobei aber auch sie bestätigt, dass viele medizinische Rezepte und Rezeptsammlungen Autorinnen zugeschrieben wurden unter anderem stamme die Mehrheit der umfangreichen Rezeptsammlung Palatina von Frauen. ${ }^{299}$ Rankin untersuchte in ihrer Studie mit dem Titel Panaceia's Daughters den medizinischen Austausch adeliger Frauen im deutschsprachigen

Greyerz, Kaspar von; Flubacher, Silvia; Senn, Philipp (Hg.): Wissenschaftsgeschichte und Geschichte des Wissens im Dialog - Connecting Science and Knowledge. Schauplätze der Forschung - Scenes of Research. Göttingen: V\&R Unipress, 2013. Zu dieser Unterteilung siehe u.a. Zedelmaier: Wissensordnungen der Frühen Neuzeit, 2007, S. 835. Siehe dazu auch Kapitel 1.4.1 zur Wissens- und Wissenschaftsgeschichte.

$296 \mathrm{Zu}$ handschriftlichen Rezeptbüchern siehe u. a. Leong: Collecting Knowledge for the Family, 2013; Leong, Elaine; Pennell, Sara: Recipe Collections and the Currency of Medical Knowledge in the Early Modern ,Medical Marketplace', in: Jenner, Mark S.R.; Wallis, Patrick (Hg.): Medicine and the Market in England and its Colonies, c. 1450-c. 1850. London: Palgrave, 2007, S. 133-152; Leong, Elaine; Rankin, Alisha Michelle (Hg.): Secrets and Knowledge in Medicine and Science, $1500-1800$ (= The History of Medicine in Context). Farnham: Routledge, 2011; Knoppers, Laura Lunger: Opening the Queen's Closet: Henrietta Maria, Elizabeth Cromwell, and the Politics of Cookery, in: Renaissance Quarterly 60, 2007, S. 464-499; Laroche, Rebecca: Medical Authority and Englishwomen's Herbal Texts, 1550-1650. Farnham: Ashgate, 2009; Pugliano, Valentina: Specimen Lists: Artisanal Writing or Natural Historical Paperwork?, in: Isis 103 (4), 2012, S. 716 726; Rankin: Panaceia's Daughters, 2013; Sorbello Staub, Alessandra: Die Basler Rezeptsammlung. Studien zu spätmittelalterlichen deutschen Kochbüchern (= Würzburger medizinhistorische Forschungen, 71). Würzburg: Königshausen \& Neumann, 2002; Tebeaux: Women and Technical Writing, 1475-1700, 1997.

297 Siehe u. a. Stine, Jennifer K.: Opening Closets: The Discovery of Household Medicine in Early Modern England, Dissertation, Stanford University, 1996; Leong, Elaine: Medical Recipe Collections in Seventeenth-Century England: Knowledge, Text and Gender. Unveröffentlichte Dissertation. Oxford University, 2006; Dies.: Recipes and Everyday Knowledge, 2018; Leong, Rankin: Secrets and Knowledge in Medicine and Science, 1500 -1800, 2011; Rankin: Panaceia’s Daughters, 2013.

298 Green, Monica Helen: The Possibilities of Literacy and the Limits of Reading: Women and the Gendering of Medical Literacy, in: Dies. (Hg.): Women's Healthcare in the Medieval West. Texts and Contexts (= Variorum Collected Studies Series). Aldershot: Routledge, 2000, S. 1-76, hier S. 46. Siehe auch Floyd, Janet; Forster, Laurel: The Recipe Reader. Narratives, Contexts, Traditions Traditions. Lincoln, Neb.: University of Nebraska Press, 2010, S. 5.

299 Rankin: Panaceia's Daughters, 2013, S. 62, 69. 
Raum, bei dem Rezepte eine bedeutende Rolle spielten. Diese Frauen hätten sich, etwa durch intensiven Austausch, medizinische Autorität erarbeiten können. ${ }^{300}$ Dies bestätigen viele Widmungsschreiben von Rezeptbüchern an Frauen: Auch einige Dedikationen Johann Jacob Weckers waren an Frauen adressiert. ${ }^{301}$ Frauen waren nicht nur für die Produktion, den Transfer und die Zirkulation von handschriftlichen Rezepten von Bedeutung, sondern auch von gedruckten. ${ }^{302}$ Ein Beispiel dafür ist Anna Wecker - die Ehefrau Johann Jacob Weckers -, unter deren Namen das Ein koestlich new Kochbuch erschienen ist. ${ }^{303}$ Neben der Erstellung

300 Ebd., u.a. S. 45.

301 U. a. Wecker: Kunstbuch deß wolerfaren Herren Alexij Pedemontani, 1569; Wecker: Kunstbůch Des Wolerfarnen Herren Alexij Pedemontani von mancherley nutzlichen vnnd bewerten Secreten oder Künsten [...] (Der Ander theil.), 1573; Wecker: Ein nutzliches Büchlein, 1569; Wecker: Artzney Buch, 1575. Auch andere mittelalterliche und frühneuzeitliche Texte wurden Frauen gewidmet, so soll etwa das The Recuyell of the Historyes of Troye (Bruges, 1473-1474) an Margarete von Burgund (1290-1315) gewidmet worden sein. [Anonym]: The Recuyell of the Historyes of Troye. Bruges, 1573-1474. Rare, Versh PQ1570.A7 E5 1892. Vgl. Coldiron: Women in Early English Print Culture, 2010, S. 65. Dazu siehe auch Kapitel 4.5, Widmungen schreiben.

302 Rankin: Panaceia's Daughters, 2013, u.a. S. 45.

303 Wecker, Anna: Ein Köstlich new Kochbuch: Von allerhand Speisen, an Gemüsen, Obs, Fleisch, Geflügel, Wildpret, Fischen vnd Gebachens. Nicht allein vor Gesunde, sondern auch vnd fürnemblich vor Kranke [...]. Amberg: Forster, 1597. Anna Wecker, geborene Keller, wurde wohl vor 1536 geboren und war die Tochter des Tuchhändlers Clemens Keller (1488-1536) und seiner vermutlich zweiten Ehefrau Anna - oder aber seiner dritten Gattin Katharina Lombard (15311559). Für erstere Möglichkeit spräche der Name Anna, da das Benennen von Kindern nach den elterlichen Namen zu dieser Zeit verbreitet war.Vgl.van Dülmen, Richard: Kultur und Alltag in der frühen Neuzeit. München: Beck, 2005 , S. 86. Für zweitere spräche die Tatsache, dass Isaak Keller (1530 - 1596), ihr Bruder, im „Heidelberger Gelehrtenlexikon“ als Stiefsohn von Simon Grynæus bezeichnet wird, den Katharina Lombard nach dem Tod von Clemens Keller 1536 wahrscheinlich im September 1538 geheiratet hat. Drüll, Dagmar: Art. „Grynaeus (Griner), Simon [d.Ä.]“, in: Dies. (Hg.): Heidelberger Gelehrtenlexikon 1386-1651, Band 3. Berlin, Heidelberg: Springer, 2002, S. 190. Anna Keller hatte wohl mindestens vier Geschwister: Thomas Keller (1516-1571), Melchior Keller (gest. 1587), Isaak Keller (1530 -1596) und Hans Jacob Keller (1531-1603). Ob es weitere Kinder gab, ist unklar. Stroux, Ulrich: Auszug Stamm Keller. Online unter: http://www.stroux.org/ patriz_f/stQV_f/Ke_f.pdf [23.02.2021]. Anna Keller heiratete ungefähr in den 1550er Jahren den Nürnberger Stadtschreiber Israel Aeschenberger. Aus dieser Ehe ging eine Tochter, Katharina, hervor. Letztere heiratete 1572 den Mediziner und Philosophen Nikolaus Taurellus, der ebenfalls an der Weckersche Buchproduktion beteiligt war. Nach dem Tod Aeschenbergers ehelichte Anna Keller Johann Jacob Wecker, wobei sie nach dessen Tod zu ihrer Tochter und Nikolaus Taurellus nach Altdorf bei Nürnberg zog, wo sie bis zu ihrem Lebensende 1596 oder 1597 lebte. Classen, Albrecht: Der Liebes- und Ehediskurs vom hohen Mittelalter bis zum frühen 17. Jahrhundert (= Volksliedstudien, Bd. 5). Münster, New York, München, Berlin: Waxmann, 2005, S. 295; Hieronymus: Sprache und Übersetzung im Basler Buchdruck, 2003, S. 57; Daems, Willem F.: 134a. Die Baslerin Anna Wecker und ihr Kochbuch von 1597^, in: Schweiz. Apoth.-Ztg./J. suisse de phar- 
dieses Buches war sie auch als Botin in die Textproduktion ihres Mannes involviert und arbeitete nach seinem Tod direkt an der Produktion Weckerscher Drucke mit. ${ }^{304}$

Die These, dass die Rezeptbuchproduktion „distinctly feminine“ gewesen sei, lässt sich meines Erachtens nicht halten, bedenkt man, dass neben diesen von Frauen gesammelten und aufgeschriebenen Rezepten auch viele Rezeptsammlungen von Männern überliefert sind. ${ }^{305}$ Man könnte vermuten, dass Frauen für Manuskripte und Männer für Drucke Rezepte sammelten; die Quellen sprechen jedoch dagegen. Ein Beispiel für ein Manuskript, das einem Mann zugeschrieben wird, ist jenes von Ludwig VI. von der Pfalz. ${ }^{306}$ Das Ein koestlich new Kochbuch hingegen wurde unter dem Namen Anna Weckers publiziert. ${ }^{307}$ Trotz der Tatsa-

macie 116 (5), 1978, s.p. Unter ihrem Namen wurden mehrere Werke veröffentlicht: Wecker, Anna: Ein Hochzeit Spruch zu Ehren vnd gluecklicher Wolfart. DemErbarn vnd Vesten Junckern Jacob Poemern vnd seiner Erbarn vnd Tugentsamen Braut Jungfrawen Barbara Loeffelholtzin: Gestellet Durch Anna Kellerin: Doctor Hannß Jacob Wecker seligen hinderlassene Wittfraw. Nürnberg: Nikolaus Knorr, 1586 sowie Dies.: Ein Köstlich new Kochbuch, 1597; Dies.: Ein Köstlich new Kochbuch Von allerhand Speisen, an Gemüsen, Obs, Fleisch, Geflügel, Wildpret, Fischen und Gebachens : Nit allein vor Gesunde: sondern auch und fürnemlich vor Krancke [...]. Amberg: Forster, 1598. Diese Ausführungen basieren auf: Zweifel, Simone: ,Communitas epistolaria‘ am Oberrhein, das Ärzte-Ehepaar Anna (gest. 1596/97) und Johann Jacob Wecker (1528-1586/88) und ihr Umfeld. Unveröffentlichte Masterarbeit. Universität Basel. Basel, 2012, Glossar.

304 Sie war z. B. direkt an der Publikation Wecker, Johann Jacob: Antidotarium speciale. Ex opt. avthorvm tam veterum quàm recentiorum scriptis fideliter congestum, methodicè digestum, \& ampliùs triente auctum. Basileae: Episcopius, 1588 beteiligt.

305 Green: The Possibilities of Literacy and the Limits of Reading: Women and the Gendering of Medical Literacy, 2000, hier S. 46. Siehe auch Floyd, Forster: The Recipe Reader, 2010, S. 5; Kavey: Books of Secrets, 2007, insbesondere Kapitel 4 sowie Staindl, Balthasar: Ein künstlichs und nützlichs Kochbuch, vormalems nie so leicht Mannen und Frawen personen von jnen selbs zulernen in Truck verfaßt [...]. Augsburg: Otmar, Valentin, 1552; Brunfels, Otto von: Von allerhandt apothekischen Confectionen, Lattwegen, Oel, Pillulen, Traencken, Trociscen, Zucker scheiblein, Salben vnnd Pflastern etc. [...]. Frankfurt am Main: Hermann Gulferich, 1552; Huber, Bartholomaeus: New Speisebůchlein. Darinnen kurtzer Vnterricht von Essen vnd Trincken / Auch von allerley Speisen vnd Getranck / so zur Menschlichen nahrung dienlich / vnd bey den Teutschen vornemlich in tăglichem Gebrauche sind / sampt vielen guten Hausartzneyen / den Einfăltigen zu gut angezeigt wird. [...]. Erfurt: Johann Beck, 1588; Platina, Bartholomaeus: Von allen Speisen und Gerichten, Koch und Kellerey. Straßburg: [s.typ.], 1530; Rumpolt, Marx: Ein new Kochbuch. Das ist Ein gründtliche beschreibung wie man recht und wol [...] allerley Speiss [...] Kochen und zubereiten solle [...]. Franckfort: Feyerabend, 1581; Ryff, Walther Hermann: New kochbuch Für die Krancken. [s.l.]: [s.typ.], 1555.

306 Von der Pfalz, Ludwig VI.: Rezeptsammlung - Universitätsbibliothek Heidelberg, Cod. Pal. germ. 192. [Amberg] 1570, Universitätsbibliothek Heidelberg, Bibliotheca Palatina - digital, Signatur: Cod. Pal. germ. 192.

307 Wecker: Ein Köstlich new Kochbuch, 1598. 
che, dass Rezeptbücher meines Erachtens nicht „distinctly feminine“ sind, sind diese Forschungsarbeiten zu Rezeptbüchern äusserst bedeutsam: Sie haben nicht nur verdeutlicht, wie wichtig Frauen für die frühneuzeitliche Medizin waren, sondern auch, dass sie in der Verbreitung von Rezepten eine bedeutende Rolle spielten. Zudem haben sie Rezeptbücher ins Blickfeld der Forschung gerückt. ${ }^{308}$

$\mathrm{Zu}$ den Rezeptbüchern zählen meines Erachtens auch Books of Secrets. Dies, da sie Anleitungen zur Herstellung von Produkten enthalten, was nach meinem Verständnis Rezeptbücher ausmacht. ${ }^{309}$ Für William Eamon unterscheiden sich Books of Secrets von Rezeptbüchern dadurch, dass erstere mehr alchemistische Rezepte enthalten und das Ziel verfolgten, Geheimnisse der Natur (,secrets of nature“) aufzudecken. ${ }^{310}$ Meines Erachtens war es jedoch nicht unbedingt die Suche nach den Geheimnissen die Natur, durch die sich Books of Secrets von anderen Rezeptbüchern unterscheiden, sondern die Tatsache, dass sie breitere Wissensbestände, etwa zu Wind und Wetter, enthalten konnten. ${ }^{311}$ Bestes Beispiel dafür ist das De Secretis libri XVII, das Wissensbestände unterschiedlichster Provenienz beinhaltet. ${ }^{312}$ Dennoch war die Verknüpfung von Wissen mit Mysteriösem und Unbekanntem nicht unbedeutend. So findet sich die Suche nach Schätzen der Natur auch in der Weckerschen Edition De Secretis libri XVII:

Unerschöpft sind die Schätze der Natur, und es versteckt sich ohne Zweifel das meiste, das im Fortlauf der Zeit durch die Bemühungen scharfsinniger Menschen ausfindig gemacht wird. ${ }^{313}$

308 Siehe u.a. Leong: Collecting Knowledge for the Family, 2013; Leong, Pennell: Recipe Collections and the Currency of Medical Knowledge in the Early Modern ,Medical Marketplace‘, 2007; Leong, Rankin: Secrets and Knowledge in Medicine and Science, 1500-1800, 2011; Knoppers: Opening the Queen's Closet: Henrietta Maria, Elizabeth Cromwell, and the Politics of Cookery, 2007; Laroche: Medical Authority and Englishwomen's Herbal Texts, 1550 - 1650, 2009; Pugliano: Specimen Lists: Artisanal Writing or Natural Historical Paperwork?, 2012; Rankin: Panaceia's Daughters, 2013; Sorbello Staub: Die Basler Rezeptsammlung; Tebeaux: Women and Technical Writing, 1475-1700, 1997.

309 Siehe auch Leong: Recipes and Everyday Knowledge, 2018, S. 4.

310 Eamon: How to Read a Book of Secrets, 2011, S. 35.

311 Zur Kategorie des „Rezeptwissen“ siehe Leong: Recipes and Everyday Knowledge, 2018, S. 4. Zu gelehrtem Wissen siehe Renn: From the History of Science to the History of Knowledge - and Back, 2015; Von Greyerz, Flubacher, Senn: Einleitung, 2013, S. 9.

312 Wecker: De Secretis libri XVII, 1582.

313 Ebd., [S. 11]. „Inexhaustus est naturæ thesaurus, et de litescunt proculdubio plurima, quæ temporis successu sagacium hominum conatibus eruentur." Siehe auch Eamon: Science and the Secrets of Nature, 1996, S. 276. 
Dieses Zitat illustriert, dass man sich auf die Suche nach diesen Geheimnissen machen muss, um sie zu entdecken. Diese Art und Weise des Erforschens wird von William Eamon als „Science as a venatio“ benannt. ${ }^{314}$ Die Books of Secrets hätten dazu beigetragen, die Geheimnisse der Natur fassbar zu machen und diese besser zu verstehen und hätten der Vorstellung der Natur den „Schleier des Mysteriösen“ genommen. ${ }^{315} \mathrm{Ob}$ das tatsächlich das Ziel war, oder ob diese Rhetorik nicht vielmehr die Bücher gerade erst interessant machen sollte, ist schwierig $\mathrm{zu}$ beurteilen - wahrscheinlich trifft beides zu. Dass diese Rhetorik die Bücher auch bewerben sollte, darauf deutet unter anderem die Tatsache hin, dass sie nach Pamela Smith auch von sogenannten Scharlatanen verwendet wurde, die auf Märkten medizinische Produkte verkauften; das Geheime des Rezepts oder des Medizinprodukts sollte dieses als besonders wertvoll erscheinen lassen und diente demnach als Verkaufsargument. ${ }^{316}$ Wie eine solche Werbung aussehen konnte, illustriert das Titelblatt des Eighteen Books of the Secrets (Abb.5). Das darin enthaltene Wissen wird hinter einem Vorhang verborgen, der zuerst geöffnet werden muss. ${ }^{317}$

„Science as a venatio“ scheint hier folglich nicht nur eine Art, Wissen zu sammeln gewesen zu sein, sondern auch ein Werbemittel. Die Weckersche Ausgabe zeigt, dass trotz der Tatsache, dass sie zu grossen Teilen aus Textinhalten bestehender Bücher besteht - das darin enthaltene Wissen also nicht geheim ist der Topos des Geheimnisses aufrecht erhalten bleibt. Das Geheimnis muss demnach nicht geheim sein, um als solches beworben zu werden. Im Gegenteil: geworben wurde gerade damit, dass Geheimnisse durch diese Bücher offengelegt wurden. ${ }^{318}$

Diese Texte beinhalten demnach offenes Geheimes und waren, so William Eamon, Teil der „popular literature“. ${ }^{319}$ Andere Forschende teilen diese Meinung. ${ }^{320}$ Bei dieser Zuschreibung wird jedoch die Diversität der Inhalte dieser

314 Eamon: Science and the Secrets of Nature, 1996, insbesondere Kapitel EIGHT.

315, „[...] the veil of mystery [...].“ Eamon: How to Read a Book of Secrets, 2011, S. $36 \mathrm{f}$.

$316 \mathrm{Zu}$ ersterer Verbindung siehe Smith: What is a Secret?, 2011, S. 47. Zu „Scharlatanen“ siehe: Gentilcore: Healers and Healing in Early Modern Italy, 1998; Ders.: „Charlatans, Mountebanks and Other Similar People“, 1995.

317 Wecker, Read: Eighteen Books of the Secrets of Art \& Nature, 1660.

318 Vgl. Kassell, Lauren: Secrets Revealed: Alchemical Books in Early-Modern England, in: History of Science 49 (162), 2011, S. 61-87.

319 Siehe u.a. Eamon: Science and Popular Culture in Sixteenth Century Italy, 1985, S. 474; Eamon: How to Read a Book of Secrets, 2011, S. 24.

320 Siehe u. a. Kavey: Books of Secrets, 2007, S. 3; Fissell, Mary E.: Popular Medical Writing, in: Raymond, Joad (Hg.): The Oxford History of Popular Print Culture. Oxford [etc.]: Oxford University 


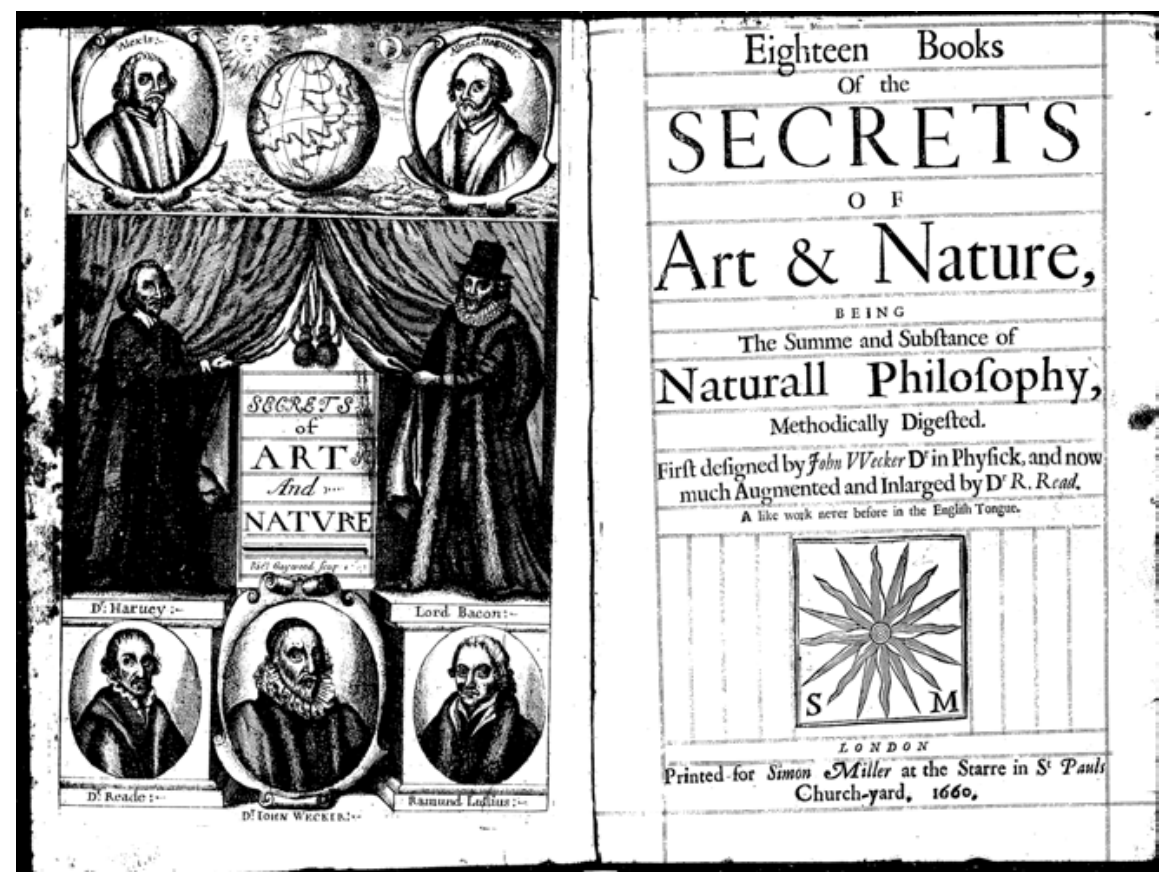

Abbildung 5: Wecker, Johann Jacob; Read, R.: Eighteen Books of the Secrets of Art \& Nature [...], 1660 .

Bücher sowie weitere Aspekte der Books of Secrets vernachlässigt, die nicht als „populär“ begriffen werden können. Im folgenden Unterkapitel soll aufgezeigt werden, warum Books of Secrets nicht als „popular literature“ verstanden werden sollten und dass sie, ganz im Gegenteil, prädestiniert waren, Wissensbestandteile unterschiedlichster Provenienz zusammenzubringen.

\subsection{Books of Secrets - „popular literature“?}

Die Kategorie „popular literature“ ist eng mit jener der „popular culture“ verknüpft. ${ }^{321}$ Laut Peter Burke entstand dieser Terminus im 18. Jahrhundert:

Press, 2011, S. 418-431, hier S. 422f.; Gadebusch Bondio: Fragmente einer weiblichen Wissenschaftsgeschichte, 1996, S. 126.

321 Damit verknüpft ist der Begriff der Kultur, den Peter Burke wie folgt definiert: „[...] a system of shared meanings, attitudes and values, and the symbolic forms (performances, artifacts) in which they are embodied“. Ders.: Popular Culture between History and Ethnology, in: Ethnologia 
This sense of contrast between two cultures had previously been expressed by the learned in references to popular culture as a collection of 'superstitions' or 'old wives' tales' (fabulae aniles), or, [...] 'little traditions of the people' (populares traditiunculae). ${ }^{322}$

Damit war dieser Unterscheidung von Beginn weg eine Hierarchisierung inhärent, die sich bis heute in den meisten Konzepten von „popular culture“ wiederfindet. $^{323}$

Die „popular culture“ oder „culture populaire“ wurde in den 1960er Jahren zum Forschungsthema der Geschichtswissenschaften, insbesondere im französischsprachigen Raum. ${ }^{324}$ Für Robert Mandrou, einem der wichtigsten Vertreter dieser Forschung, handelte es sich dabei um eine vom „Volk“ hervorgebrachte Kultur, eine Kultur, die als authentisch betrachtet wurde. ${ }^{325}$ Dieses Verständnis ist mehrfach problematisch, nicht nur, weil vom „Volk“ gesprochen wird, sondern auch, da es unmöglich ist zu wissen, ob ein Text authentisch ist, ob er also vom „Volk“ stammt oder nicht. ${ }^{326}$ Hinter der Einführung dieses Feldes stand laut Do-

Europaea. Journal of European Ethnology Volume XIV, 1984, S. 5-13, hier S. 5. In der Forschung finden sich unzählige Begriffe von Kultur. Auf dieses Feld wird hier nicht weiter eingegangen, da es den Umfang der vorliegenden Arbeit sprengen würde. Zu Kulturbegriffen siehe u.a. Busche, Hubertus: Was ist Kultur? Die vier historischen Grundbedeutungen, in: Dialektik. Zeitschrift für Kulturphilosophie 1, 2000, S. 69-90; Schröder, Gerhart; Breuninger, Helga (Hg.): Kulturtheorien der Gegenwart. Ansätze und Positionen. Frankfurt am Main; New York: Campus-Verl., 2001; Bachmann-Medick, Doris: Cultural Turns. Neuorientierungen in den Kulturwissenschaften (= Rororo, 55675). Reinbek bei Hamburg: Rowohlt, 2006.

322 Burke: Popular Culture between History and Ethnology, 1984, S. 6.

323 Siehe Storey, John: Cultural Theory and Popular Culture. An Introduction. Harlow, England, New York: Prentice Hall, 2001³, S. 1, 14. Eine solche Hierarchisierung findet sich u. a. bei Burke, Peter: Popular Culture in Early Modern Europe. New York: New York University Press, 1978, Prologue.

324 Kalifa, Dominique: Les historiens français et „le populaire“, in: Hermès 42, 2005, S. 54 - 59, hier S. 54. Hier sind insbesondere die Arbeiten von Robert Mandrou und Robert Muchembled zu nennen: Mandrou, Robert: De la culture populaire aux XVIIe et XVIIIe siècles. La bibliothèque bleue de Troyes. Paris: Stock, 1975; Muchembled, Robert: Culture populaire et culture des élites dans la France moderne : XVe-XVIIIe siècle (= Champs). [Paris]: Flammarion, 2011.

325 „La culture populaire est restée, encore maintenant, pour tout un courant de pensée qui pourrait être appelé populiste, la culture qui est l'œuvre du peuple [...] cette définition se situe en son fond sur le plan de la création, artistique ou littéraire : à la limite ne mériterait à ses yeux le nom de culture populaire que l'œuvre authentiquement créée par les gens du peuple (au sens étroit de ce terme), l'œuvre élaborée par lui.“ Mandrou: De la culture populaire aux XVIIe et XVIIIe siècles, 1975, S. 11.

326 Zum Volksbegriff und dessen Problematik siehe Deiters, Franz-Josef: Volk, in: Ueding, Gert (Hg.): Historisches Wörterbuch der Rhetorik. Tübingen: De Gruyter, 2009, Sp. 1164-1171; Link, Fabian: Peuple (Volk) et race (Rasse), in: Christin, Olivier; Barat, Raphaël (Hg.): Dictionnaire des concepts nomades en sciences humaines. Paris: Métailié, 2010, S. 71-85. 
minique Kalifa kein spezifisches methodologisches Programm; vielmehr ging es darum, den Blick auf bislang kaum betrachtete Akteurinnen und Akteure zu lenken. ${ }^{327}$ Dazu zählten von der Gesellschaft Ausgeschlossene wie etwa Kriminelle oder Deviante, aber auch Arme, Frauen, junge Menschen, Bauern - alle, die nicht Teil der „Elite“ waren. ${ }^{328}$ Durch diese Aufteilung entsteht eine Dichotomie, weshalb Peter Burke berechtigt fragt:

But who are the people? [...] For the clergy, the people are the laity ; for the nobility, the commoners; for the rich, the poor; and for those of us who have been to university, it is obvious that the people are those who lack degrees. ${ }^{329}$

Die Zuschreibung zu „Elite“ oder „Volk“ ist demnach immer abhängig von der Perspektive, vom Kontext und auch von der Person selbst, die diese Zuschreibung vornimmt. ${ }^{330}$ Aus diesem Grund - und weil sie unterschiedlich, ja gar gegensätzlich gefüllt werden kann - hält John Storey die Kategorie „popular culture“ gar für eine leere konzeptuelle Kategorie. ${ }^{331}$

Eine andere Definition von „popular culture“ bezieht sich auf die grosse Anzahl von Menschen, die etwas mögen, was dieses dann „populär“ macht, etwa in Bezug auf Musik. ${ }^{332}$ Hier wiederum stellt sich die Frage, wie gross diese Anzahl von Menschen sein muss, damit etwas „populär“ wird. Zudem wird auch hier von der „Hochkultur“ abgegrenzt in dem Sinne, dass alles „Populärkultur“ ist, was nicht „Hochkultur“ ist. ${ }^{333}$ Dieses Begriffsverständnis kommt nahe an die Definition von Peter Burke, der schreibt: „As for popular culture, it is perhaps best defined initially in a negative way as unofficial culture, the culture of the nonelite, the ,subordinate classes ' [...]. “334 Burke schlägt vor, die unterschiedlichen Kulturen weiter aufzuteilen in Subkulturen, unterteilt nach Raum, Sprache, lite-

327 Kalifa: Les historiens français et „le populaire“, 2005, S. 54.

328 Ebd., S. 55.

329 Burke: Popular Culture between History and Ethnology, 1984, S. 5.

330 Storey: Cultural Theory and Popular Culture, 2001, S. 10.

331 Ebd., S. 1. Zu unterschiedlichen „Befüllungen“ des Begriffs siehe u. a. Hall, Stuart: Notes on Deconstructing ,the Popular, in: Storey, John (Hg.): An Introduction to Cultural Theory and Popular Culture. Athens: University of Georgia Press, 1998, S. 442-453, hier S. 446; Gans, Herbert J.: Popular Culture and High Culture. An Analysis and Evaluation of Taste. New York: Basic Books, 1999²; Parker, Holt N.: Toward a Definition of Popular Culture, in: History and Theory 50 (2), 2011, S. 147-170, hier S. 148; Cole, Richard G.: In Search of a New Mentality: The Interface of Academic and Popular Medicine in the Sixteenth Century, in: The Journal of Popular Culture 26 (4), 1993, S. 155 - 172, hier S. 155; Gentilcore: Was There a „Popular Medicine“ in Early Modern Europe, 2004. 332 Storey: Cultural Theory and Popular Culture, 2001, S. 6.

333 Ebd.

334 Burke: Popular Culture in Early Modern Europe, 1978, Prologue. 
rarischer Tradition etc. ${ }^{335}$ Eine dieser Kulturen, die „Elitekultur“, zeichne sich laut Peter Burke in der Frühen Neuzeit dadurch aus, dass deren Mitglieder über Lateinkenntnisse verfügten. Weiter unterscheidet er zwischen einer Gruppe, die schreiben und lesen konnte, einer, die diese Fähigkeiten zum Teil beherrschte („semi-literacy“), und einer dritten Gruppe, bei der weder Schreib- noch Lesefähigkeit vorhanden war. ${ }^{336}$ Diese Ausdifferenzierung erscheint auf den ersten Blick gut auf die Frühe Neuzeit anwendbar. Doch: Wie können wir die Lese- und Schreibfähigkeit einer frühneuzeitlichen Person beurteilen, wenn wir nicht über entsprechende Quellen verfügen? Wo fangen Lese- und Schreibfähigkeit an und wie werden sie gemessen? ${ }^{337}$ Peter Burke würde wohl argumentieren, dass er die Kategorien eher als fluide denn als fest und Kultur als ein offenes und wenig definiertes System betrachte. Zudem argumentiert er, dass sich die Kulturen gegenseitig beeinflussten: Nicht nur Top-down, sondern auch Bottom-up. ${ }^{338}$ Auch diesem Modell bleibt folglich die Hierarchie zwischen den unterschiedlichen Kulturen inhärent. Nach Bob Scribner hängt die Rechtfertigung solcher ,two-tier‘Modelle von folgender Annahme ab: „[Popular culture, SZ] was the culture of the peasantry and other subordinate social groups, all of which were held by many educated elites to be lacking in ,culture'. This is the reality behind, and the justification for, the ,two-tier“ model.“339 Folglich ist es der „Mangel an Kultur“, welcher der „Populärkultur“ zugeschrieben wird. Das erinnert wieder an frühere Definitionen, in denen „populär“ mit deviant oder „abergläubisch“ verbunden wurden. Solche Abwertungen scheinen so stark mit der Geschichte des Begriffs „populär“ verwoben zu sein, dass wir uns kaum davon lösen können. Doch es ist nicht die Abwertung, die den Begriff „populär“ für frühneuzeitliche Rezepte wenig brauchbar macht, sondern einerseits die Tatsache, dass er kaum zu fassen ist, wie es John Storey deutlich gemacht hat, andererseits, dass Rezeptbüchern so viele unterschiedliche Elemente inhärent sind, dass eine Zuschreibung zu „populär“ zu vereinfachend wäre. Welche Elemente ich meine, möchte ich im Folgenden kurz ausführen.

335 Ebd., S. $23 \mathrm{f}$.

336 Ebd., S. 63.

337 Siehe auch Chartier, Roger: Chapter IX. Culture as Appropriation: Popular Cultural Uses in Early Modern France, in: Kaplan, Steven L. (Hg.): Understanding Popular Culture. Europe from the Middle Ages to the 19th Century (= New Babylon). Berlin: Mouton, 1984, S. 229-254, hier S. 236. 338 Ebd., S. 29; Burke: Popular Culture between History and Ethnology, 1984, S. 5; Ders.: Popular Culture in Early Modern Europe, 1978, hier S. 23 f., 58.

339 Scribner, Bob: Is a History of Popular Culture Possible?, in: History of European Ideas 10 (2), 1989, S. 175-191, hier S. 181. 
Ein Kriterium, anhand dem Eamon die Books of Secrets der „popular literature“ zuschreibt, ist jenes der Autorschaft. Er argumentiert, dass die „Professors of Secrets“ nicht zum akademischen Establishment gehörten. ${ }^{340}$ Dies trifft für Johann Jacob Wecker jedoch nicht zu: Er hatte in Basel, Wittenberg und Bologna studiert und promoviert, war später Professor für Logik und Latein an der Universität Basel und amtete als Dekan der medizinischen Fakultät, bevor er Stadtarzt von Colmar wurde. ${ }^{341}$ Er war vernetzt mit anderen Professoren, etwa mit Theodor Zwinger, und befand sich keinesfalls ausserhalb des akademischen Establishments. Diese Verbindung zur „popular literature“ muss bei Wecker demnach ausgeschlossen werden. Des Weiteren waren neben Wecker weitere Personen und Elemente in die Produktion seiner Bücher involviert. Diese entstammten unterschiedlichen Schichten und waren unter anderem ebenfalls Teil des akademischen Establishments. Zu nennen ist hier wiederum Theodor Zwinger, der eng mit Wecker zusammengearbeitet hat.

Doch waren die Autoritäten, aus denen Wecker kompiliert hat, „populär“ im Sinne einer Burkeschen „Populärkultur“? Nein, im Gegenteil: Sie waren äusserst variantenreich und können nicht in eine Kategorie eingeteilt werden. Denn Wecker und seine Kollaborierenden versammelten insbesondere für die De Secretis libri XVII aus dem Jahr 1582 Wissensbestände aus verschiedenen Wissenstraditionen, Zeiten und Räumen. ${ }^{342}$ Selbiges wird auch in der Vorrede des Alessio Piemontese behauptet, die Wecker 1571 übersetzte. Darin steht:

Und während ich stets bemüht war, über die Weisheit und heimliche verborgene natürliche Künste zu lernen, bin ich siebenundfünfzig Jahre hin und her, an viele Orte der Welt gezogen, um viele gelehrte Männer kennen zu lernen. Dabei habe ich nicht allein von gelehrten und anderen grossen Herren, sondern auch von Weibern, Handwerkern und Bauern viele und mancherlei heimliche und verborgene Künste erlangt und bekommen. ${ }^{343}$

Hier wird betont, dass nicht nur Wissen von Gelehrten, sondern auch von „grossen Herren“, Frauen, Werkleuten und Bauern verwendet worden sei. Diese

340 Eamon: Science and the Secrets of Nature, 1996, S. 135.

341 Siehe Jöcher: Allgemeines Gelehrten Lexicon, col.1839; Hartmann, Jenny: Die Briefe aus den Jahren 1544-1547, 1967, S. 170; Jenny: 1553-30. Juni 1554, 1982, S. 37; Rocchietta: Storia della medicina, S. 879.

342 Wecker: De Secretis libri XVII, 1582b.

343 „Vnd dieweil ich allzeit von natur / mich der weißheit / vnd heimlichen verborgnen natürlichen künsten zůlernen / geflissen / bin ich siben vnd fünftzig jar hin vnd haer / an vil ort der welt gezogen / damit ich vil gelerte menner erkennen moechte. Derhalben hab ich nit allein von gelerten / vnd anderen grossen herren / sonder auch von weybern / werckleüten vnd bauren vil vnd mancherley heimliche vnd verborgne kuenst erlangt vnd vberkommen." Wecker, Johann Jacob: Kunstbůch Des Wolerfarnen Herren Alexij Pedemontani, 1571, [S. 16]. 
Vielheit würde negiert, würde man diese beiden Texte als „Populärliteratur“ verorten.

Neben den Inhalten lässt sich fragen, ob allenfalls die Leserschaft als Teil der „popular culture“ bezeichnet werden könne. Analysiert man die Lesespuren der Weckerschen Publikationen, so wird deutlich, dass sich Annotationen auf Lateinisch, ja sogar auf Griechisch finden lassen, was auf ein gelehrtes Publikum hindeutet. ${ }^{344}$ Auf unterschiedliche Weisen gebildet waren die mir bekannten Besitzer Weckerscher Publikationen: Bislang konnten ein Markgraf, ein Theologe und ein Chirurg als Besitzer identifiziert werden. ${ }^{345}$ Sie entstammten unterschiedlichen Schichten und hatten verschiedene Bildungsniveaus, was wiederum gegen die von Eamon verwendete Kategorie „populär“ spricht. Ebenfalls nicht „populär“ waren die Adressaten der Weckerschen Übersetzung der De Secretis aus dem Jahr 1571: „Wann in disem ersten bůch beschreibt vnd leret er [Piemontese, SZ] mancherley gůte artzneyen [...] sonderlich so man die selbe recht / vnd mit rath der gelerten artzet brauchet.“" ${ }^{346}$ Hier wurden folglich Ärzte als Adressaten benannt, die ebenfalls kaum als der „Populärkultur“ zugehörig bezeichnet werden können.

All diese Aspekte sprechen gegen eine Kategorisierung von Books of Secrets als „popular literature“. Sie zeigen auf, dass Books of Secrets auf mehreren Ebenen von Personen und Elementen verschiedener Zeiten, Räume und Schichten beeinflusst waren, weshalb eine solche Zuteilung nicht greift. Diese Diversität betrifft nicht nur die in diesen Büchern enthaltenen Wissensbestände, sondern auch die Besitzenden, die Rezipierenden sowie die Autorschaft dieser Texte: Unterschiedliche Kompilationsnetzwerke, in die verschiedene Akteurinnen und Akteure aus unterschiedlichsten Schichten involviert waren, produzierten diese Texte. Das Zusammenspiel dieser Elemente wird hier als Kompilationsnetzwerk konzeptualisiert. Was damit genau gemeint ist und welche theoretischen Vorannahmen dahinterstehen, ist Thema des folgenden Kapitels.

344 Siehe u. a.: Wecker, Johann Jacob: Medicinae vtrivsque syntaxes. Basileae: Evsebii Episcopi, et Nicolai fr. hæredum, 1576. SBB Berlin. Jl 2906; Wecker: De Secretis libri sex, 1559. Herzog August Bibliothek Wolfenbüttel. 138.9 Medica.

345 Wecker, Johann Jacob: Kunstbůch des Wolerfarnen Herren Alexij Pedemontani von mancherley nutzlichen vnnd bewerten Secreten oder Künsten, jetz neuwlich auß Weltscher vnnd Lateinischer sprach inn Teutsch gebracht [...]. Basel: Pietro Perna, 1573. SBB Berlin. 50 MA 10404; Wecker: D. Alexii Pedemontani De Secretis libri septem, 1563. BSB München. M. med. 25.

346 Wecker: Kunstbůch Des Wolerfarnen Herren Alexij Pedemontani, 1571, [p. 9]. Siehe auch Zitat S. 32. 